\title{
Screening of immunosuppressive factors for biomarkers of breast cancer malignancy phenotypes and subtype-specific targeted therapy
}

\author{
Ping Wang Equal first author, 1 , Jiaxuan Liu Equal first author, 1 , Yunlei Song ${ }^{2}$, Qiang Liu ${ }^{3}$, Chao Wang ${ }^{4}$, Caiyun Qian ${ }^{5}$, Shuhua \\ Zhang $^{6}$, Weifeng Zhu ${ }^{5}$, Xiaohong Yang ${ }^{5}$, Fusheng Wan ${ }^{5}$, Zhuoqi Liu ${ }^{\text {Corresp., }}{ }^{\text {, }}$, Daya Luo \\ ${ }^{1}$ Queen Mary School, Nanchang University, Nanchang, China \\ 2 Key Laboratory of Prevention and treatment of cardiovascular and cerebrovascular diseases of Ministry of Education, Gannan Medical University, \\ Ganzhou, China \\ 3 National Cancer Center/Cancer Hospital, Chinese Academy of Medical Sciences and Peking Union Medical College, Beijing, China \\ 4 School of Basic Medical Sciences, Nanchang University, Nanchang, China \\ 5 Department of Biochemistry and Molecular Biology, School of Basic Medical Sciences, Nanchang University, Nanchang, China \\ 6 Jiangxi Cardiovascular Research Institute, Jiangxi Provincial People's Hospital, Nanchang, China \\ 7 Jiangxi Province Key Laboratory of Tumor Pathogens and Molecular Pathology, Nanchang University, Nanchang, People's Republic of China \\ Corresponding Authors: Zhuoqi Liu, Daya Luo \\ Email address: liuzhuoqi@ncu.edu.cn, luodaya@ncu.edu.cn
}

To screen and validate immunosuppressive factors in luminal- and basal-like breast cancer cell lines and tissue samples associated with malignant phenotypes. The mRNA microarray datasets, GSE40057 and GSE1561, were downloaded and remodelled, and differentially expressed genes (DEGs) were identified. Weighted Gene Co-expression Network Analysis (WGCNA) and Gene Ontology (GO) and KEGG pathway enrichment analysis were performed to explore the immune-related events that related to the basal-like trait. The online resources, GOBO, Kaplan-Meier Plotter and UALCAN, were employed to screen for immunosuppressive factors associated with breast cancer malignant phenotypes. Immunohistochemistry was used to evaluate VEGFA and MIF levels in breast tumours and normal breast tissues; qPCR and western blot were used to validate the expression of clinical immune-oncology (IO) therapeutic targets CD274 (PD-L1) and IL8 in cell lines. The results showed that there were varies immune-related events contribute to the trait of basal-like breast cancer. First, TGF $\beta 1$ and IL8 had higher average expression levels in more malignant cell lines; Second, MIF and VEGFA had higher average expression levels in more malignant breast cancer tissue, and the high expression levels of them were associated with poor survival rate. Third, 10 targets CD274 and IL8 were confirmed that more suitable for the treatment of basal-like breast cancer. In view of the above, during the formation and development of breast cancer, immune-related genes are always activated, and immunosuppressive factors, IL8, TGF $\beta 1$, MIF and VEGFA are up-regulated. Such molecules could be used as biomarkers for breast cancer prognosis. However, because individual Peer) reviewing PDF | (2019:03:35676:1:1:CHECK 15 May 2019) 
immune-related factors can play several biological roles, the mechanistic relationship between immunosuppressive factors and breast cancer malignant phenotypes and the feasibility of their application as drug targets require further investigation. 


\section{Screening of immunosuppressive factors for}

2 biomarkers of breast cancer malignancy

3 phenotypes and subtype-specific targeted therapy

4 Ping Wang ${ }^{1}$, Jiaxuan Liu ${ }^{1}$, Yunlei Song ${ }^{2}$, Qiang Liu ${ }^{3}$, Chao Wang ${ }^{4}$, Caiyun Qian ${ }^{5}$, Shuhua Zhang ${ }^{6}$,

5 Weifeng Zhu ${ }^{5}$, Xiaohong Yang, Fusheng Wan ${ }^{5}$, Zhuoqi Liu ${ }^{5}$, Daya Luo ${ }^{5,7}$

$6 \quad{ }^{1}$ Queen Mary School, Nanchang University, Nanchang, China

$7 \quad{ }^{2}$ Key Laboratory of Prevention and treatment of cardiovascular and cerebrovascular diseases of Ministry

8 of Education, Gannan Medical University, Ganzhou, China

$9{ }^{3}$ National Cancer Center/Cancer Hospital, Chinese Academy of Medical Sciences and Peking Union

10 Medical College, Beijing, China

$11{ }^{4}$ School of Basic Medical Sciences, Nanchang University, Nanchang, China

$12{ }^{5}$ Department of Biochemistry and Molecular Biology, School of Basic Medical Sciences, Nanchang

13 University, Nanchang, China

$14{ }^{6}$ Jiangxi Cardiovascular Research Institute, Jiangxi Provincial People's Hospital, Nanchang, China

$15{ }^{7}$ Jiangxi Province Key Laboratory of Tumor Pathogens and Molecular Pathology, Nanchang University,

16 Nanchang, People's Republic of China

17 Corresponding Authors: Zhuoqi Liu, Daya Luo

18 Email address: liuzhuoqi@ncu.edu.cn, luodaya@ncu.edu.cn 


\section{Abstract}

28 To screen and validate immunosuppressive factors in luminal- and basal-like breast cancer cell

29 lines and tissue samples associated with malignant phenotypes. The mRNA microarray datasets,

30 GSE40057 and GSE1561, were downloaded and remodelled, and di $\square$ erentially expressed genes

31 (DEGs) were identified. Weighted Gene Co-expression Network Analysis (WGCNA) and Gene

32 Ontology (GO) and KEGG pathway enrichment analysis were performed to explore the immune-

33 related events that related to the basal-like trait. The online resources, GOBO, Kaplan-Meier

34 Plotter and UALCAN, were employed to screen for immunosuppressive factors associated with

35 breast cancer malignant phenotypes. Immunohistochemistry was used to evaluate VEGFA and

36 MIF levels in breast tumours and normal breast tissues; qPCR and western blot were used to

37 validate the expression of clinical immune-oncology (IO) therapeutic targets CD274 (PD-L1)

38 and IL8 in cell lines. The results showed that there were varies immune-related events contribute

39 to the trait of basal-like breast cancer. First, TGF $\beta 1$ and IL8 had higher average expression levels

40 in more malignant cell lines; Second, MIF and VEGFA had higher average expression levels in

41 more malignant breast cancer tissue, and the high expression levels of them were associated with

42 poor survival rate. Third, IO targets CD274 and IL8 were confirmed that more suitable for the

43 treatment of basal-like breast cancer. In view of the above, during the formation and

44 development of breast cancer, immune-related genes are always activated, and could be used as biomarkers for breast cancer prognosis. However, because individual immunerelated factors can play several biological roles, the mechanistic relationship between immunosuppressive factors and breast cancer malignant phenotypes and the feasibility of their application as drug targets require further investigation.

\section{Introduction}

Breast cancer, the most frequently diagnosed cancer and the second most fatal malignancy in women around the world, affects one in eight women ("Cancer facts \$figures 2016," 2016). With the advent of gene expression profiling over the last 15 years, breast cancers have been classified into luminal A, luminal B, human epidermal growth factor receptor 2 (HER2 or ERBB2)enriched, basal-like, and claudin-low categories (Liu \& Wang, 2015; Prat et al., 2015). Among these categories, basal-like breast cancer has garnered significant attention among researchers, as it accounts for $\sim 75 \%$ of the highly malignant triple-negative subtype. This biologically aggressive neoplasia takes on several malignant phenotypes, including early onset, high histological grade, increased distant recurrence and visceral metastases, insensitivity to endocrine and targeted therapy and poor prognosis (Bahnassy et al., 2015). Although biomarkers for breast 
62 cancer prognosis and therapy (Jezequel et al., 2012) have markedly improved treatment

63 decisions, inconsistent diagnostic criteria for basal-like breast cancer and controversial research

64 findings necessitate the discovery of more specific molecular markers (Tomao et al., 2015).

Malignant tumor phenotypes, such as invasiveness, metastasis, drug resistance and poor prognosis, depend on both the distinct genetic and epigenetic characteristics of the tumor as well as other factors in the tumor microenvironment (Gandellini et al., 2015). The tumor microenvironment is composed of tumor cells, various types of stromal cells and the extracellular matrix, in which tumor cells and stromal cells interact by releasing a variety of cytokines, chemokines and growth factors (M. Xu et al., 2012). In recent years, it has become clear that tumor cells, as well as other cells and factors that accumulate in tumor-bearing hosts, play a critical role in patient outcomes (Schlößer et al., 2014). On the one hand, a variety of immune cells can be induced to kill tumor cells. On the other hand, tumor cells have many strategies for escaping immune attack, including the release of immunosuppressive factors. The presence of immunosuppressive factors induces local immune escape in the tumor microenvironment, which thwarts antitumor immune responses and poses a major obstacle to many immunotherapeutic or conventional therapeutic approaches. Fortunately, the high expression levels of these immunosuppressive factors may also be studied to identify therapeutic targets. Currently, multiple immune-oncology (IO) therapeutic targets are available in clinical therapy (Szekely et al., 2018). However, due to the multifaceted functions of many immunosuppressive factors in different tumor types and stages of development, there remains controversy regarding their actual and fundamental roles in tumor pathology. This ambiguity prevents the clinical application of immunosuppressive factors as diagnostic and therapeutic biomarkers.

In this paper, by comparing gene expression patterns between basal- and luminal-like breast cancer cell lines and tissue samples, which have different levels of aggressiveness and malignancy, we sought to screen for and verify the immunosuppressive factors associated with a malignant phenotype and investigate the significance of IO targets in the clinical treatment of breast cancer. These immunosuppressive factors could be used as additional markers to identify malignant breast cancer and further tailor therapies for individual breast cancer patients.

\section{$91 \quad$ Materials and Methods}

\section{Gene Expression Microarray Analysis}

93 The expression monitoring array raw data were downloaded from the Gene Expression Omnibus 94 (GEO) database (Barrett et al., 2012) with accession numbers GSE40057 (Luo et al., 2013) and 95 GSE1561 (Farmer et al., 2005). GSE40057 included 10 breast cancer cell lines and 2 immortalized breast epithelium cell lines analyzed with the Affymetrix Human Genome U133 Plus 2.0 Array; the GSE1561 data contained 49 breast cancer tissue samples that were analyzed 
99 differentially expressed gene (DEG) screening was performed in R using Bioconductor and

100 associated packages (Gentleman et al., 2004).

101 Weighted Gene Coexpression Network Analysis (WGCNA) and Gene ontology (GO) and 102 KEGG pathway Enrichment Analysis

103 For genome-wide expression profile data of tissues and cell lines, the missing values were first

104 removed, and the genes with average expression levels less than 0.5 were filtered out. Second, all

105 samples performed well in hierarchical clustering, and no outliers needed to be removed. The

106 step-by-step method of the WGCNA package (Langfelder \& Horvath, 2008) in R was used to

107 construct the module and coexpression network. Soft thresholds were generated by the

108 pickSoftThreshold function of the WGCNA package, with tissue data set to 28 and cell line data

109 set to 10. The adjacency matrix and the topological overlap matrix (TOM) was calculated

110 according to the corresponding soft threshold. Based on TOM, the corresponding dissimilarities

111 between each gene were calculated, and 400 genes were randomly selected for TOM

112 visualization. In addition, we constructed a hierarchical cluster tree of all genes based on the

113 dissimilarity matrix. Using the dynamic tree cut method, the branches of the hierarchical cluster

114 tree were cut to identify modules. Subsequently, with the hierarchical clustering data of the

115 eigengene module data, a height cutoff of 0.25 was chosen, and similar modules were merged to

116 build the final coexpression network. Finally, the eigengene module data were visualized and

117 module-trait associations were quantified. GO and KEGG pathway enrichment analyses were

118 performed for genes in each module using the DAVID functional annotation clustering tool

119 (http://david.abcc.ncifcrf.gov) (Huang da, Sherman, \& Lempicki, 2009).

\section{GOBO analysis}

$121 \mathrm{GOBO}$ (http://co.bmc.lu.se/gobo/), an online resource with mRNA microarray profiling data

122 from 51 breast-derived cell lines (Ringner, Fredlund, Hakkinen, Borg, \& Staaf, 2011), was used

123 to validate the mRNA expression levels of four immunosuppressive factor genes identified from

124 the screened cell lines of GSE40057.

\section{UALCAN and Kaplan-Meier survival analyses}

126 UALCAN (http://ualcan.path.uab.edu/index.html), an interactive web resource that contains a

127 large amount of cancer transcriptome data derived from TCGA and MET500 transcriptome

128 sequencing (Chandrashekar et al., 2017), was used to explore the mRNA expression levels of 6

129 immunosuppressive genes identified from the screened GSE1561 tissue samples. Kaplan-Meier

130 Plotter (http://kmplot.com/analysis/), an open web-based resource (Gyorffy, Surowiak, Budczies,

131 \& Lanczky, 2013), was used to determine the relationship between the overall survival rate and

132 mRNA expression levels of 6 immunosuppressive genes identified from the screened GSE1561

133 tissue samples.

134 Immunohistochemistry (IHC) 
135 VEGFA and MIF levels in normal and breast cancer tissues were evaluated by IHC using

136 polyclonal antibodies (1:250 dilution, DF7470 and DF6404, Affinity Biosciences, Cincinnati,

137 USA) on commercial tissue arrays (Shanghai Outdo Biotech Co., Shanghai). The array consists

138 of 10 normal and 90 breast tumor specimens. Each sample was given a modified histochemical

139 score (MH score), which was assessed by both the proportion and the intensity of cells stained at

140 each intensity, to reflect the staining intensity. The intensity of each grade is the average MH

141 score of all samples in that grade.

\section{Cell culture and total RNA isolation}

143 The breast cancer cell lines MDA-MB-231 and T47D were cultured in DMEM supplemented

144 with 10\% fetal bovine serum (FBS). MCF7 cells were cultured in RPMI-1640 medium with $10 \%$

145 FBS, and BT549 cells were cultured in RPMI-1640 medium with $0.023 \mathrm{IU} / \mathrm{ml}$ insulin and $10 \%$

146 FBS. Total RNA was extracted using TRIzol ${ }^{\circledR}$ reagent (Invitrogen, Carlsbad, CA, USA).

147 qRT-PCR analysis of mRNA expression levels of CD274 and IL8

148 Two micrograms of total RNA was reverse-transcribed using the RevertAid ${ }^{\mathrm{TM}}$ First-Strand 149 cDNA Synthesis Kit (Thermo, Boston, USA). SYBR ${ }^{\circledR}$ Premix Ex Taq ${ }^{\mathrm{TM} I I}$ (TaKaRa, Shiga,

150 Japan) was used to conduct quantitative RT-PCR. The primer sequences used for RT-PCR were

151 as follows: CD274-Forward: CGTTGTGCTTGAACCCTTGA, CD274-Reverse:

152 ACACAAGGAGCTCTGTTGGA; IL8-Forward: GAGACAGCAGAGCACACAAG, IL8-

153 Reverse: TTGGGGTGGAAAGGTTTGGA; $\beta$-actin-Forward: GAACGGTGAAGGTGACAG,

154 and $\beta$-actin-Reverse: TAGAGAGAAGTGGGGTGG. Each sample was analyzed in triplicate.

155 According to the manufacturer's suggested protocols, Applied Biosystems ${ }^{\circledR} 7500$ Real-Time

156 PCR Systems (Thermo, Boston, USA) were used for the real-time PCRs. The $\Delta \Delta C t$ method was

157 used to calculate the fold change in gene expression.

158 Western blot analysis of the protein expression level of CD274 and IL8

159 Total proteins were extracted from cells in RIPA Lysis Buffer (Vazyme, Piscataway, NJ, USA)

160 containing protease inhibitors. A total of $40 \mu \mathrm{g}$ of protein from each sample was denatured,

161 fractionated by 10\% SDS-PAGE, and transferred to a PVDF membrane (Immobilon ${ }^{\circledR}-\mathrm{P}$ Transfer

162 Membrane, Millipore, Milan, Italy). After blocking nonspecific antigens with 5\% skim milk

163 solution, blots were incubated overnight at $4{ }^{\circ} \mathrm{C}$ with primary rabbit monoclonal antibodies

164 against IL8 (1:1000 working dilution, DF6998, Affinity Biosciences, Cincinnati, USA), CD274

165 (1:1000 working dilution, DF6526, Affinity Biosciences, Cincinnati, USA) or $\beta$-actin (1:1000

166 working dilution, Santa Cruz Biotechnology, Inc., Santa Cruz, CA, USA) in 5\% skim milk

$167 \quad 0.05 \%$ TBS-Tween 20 buffer. Antibody binding to the membrane was detected with a secondary

168 antibody (goat anti-rabbit IgG 1:5000, ZSGB Biosciences, Beijing, China) conjugated to

169 horseradish peroxidase and visualized by enzyme-linked chemiluminescence (EasySee ${ }^{\circledR}$

170 Western Blot Kit, TransGen Biotechnology, Beijing, China) with the Scientific MYECL Imager 
171 (Thermo, Boston, USA). Densitometric analysis performed with ImageJ software was used to

172 normalize the signals of IL8 and CD274. The intensity of the two bands was normalized against

173 the signal of $\beta$-actin.

\section{Statistical analysis}

175 Each experiment was repeated at least 3 times. DEGs were identified by the Bioconductor

176 'limma' package using moderated t-tests $(\mathrm{p}<0.05)$ and comparisons of multiple change (basal vs.

177 luminal, GSE40057>2-fold, GSE1561>1.5-fold). The association of MIF and VEGFA

178 expression and clinicopathological data were analyzed by one-way ANOVA in SPSS 17.0 (SPSS

179 Inc. Chicago, IL).

\section{Results}

\section{Identification of DEGs}

182 To study the gene expression profiles of different breast cancer cell lines and tissue-based

183 microarray datasets, GSE40057 and GSE1561 data from the GEO database were downloaded,

184 re-modeled, analyzed and compared. According to the results of the PCA (Fig. 1A), the samples

185 were successfully separated into 3 major groups: luminal-like (luminal A, luminal B), basal-like

186 and edged samples. In the hierarchical clustering (Fig. 1B), 8 cell lines (4 basal-like and 4

187 luminal-like) from GSE40057 and 32 tissue samples (16 basal-like and 16 luminal-like) from

188 GSE1561 were chosen based on the proximities between the samples for subsequent analyses.

189 The results showed that 2188 and 1963 genes were significantly differentially expressed between

190 the luminal-like and basal-like groups of cell lines and tissue samples, respectively (Table S1).

\section{WGCNA, GO and KEGG pathway Enrichment analyses of each gene module}

192 To investigate what causes the difference in the degree of malignancy between basal-like and

193 luminal-like breast cancer, the sets of genes related to basal- and luminal-like breast cancer were

194 first screened by constructing a gene coexpression network by WGCNA. Then, 25 and 6 gene

195 modules for GSE40057 (cell lines) and GSE1561 (tissue samples) were identified, respectively

196 (Fig. 2A and 2C). The member genes involved in the same module were highly interconnected

197 and further analyzed in the GO and KEGG pathway enrichment analysis. For cell lines, the light

198 yellow, light green, and dark magenta modules had significant correlations with luminal-like

199 traits $(\mathrm{p}<0.05)$; the black and orange modules had significant correlations with basal-like traits

$200(p<0.05)$ (Fig. 2B). For the tissue samples, the tan module had a significant correlation with

201 luminal-like traits ( $<<0.05$ ), and the black, midnight blue, cyan, red and gray modules had a

202 significant correlation with basal-like traits $(p<0.05)$ (Fig. 2D). Interestingly, the subsequent

203 enrichment analyses for the genes in each module showed that the enrichment results of the

204 black module in the tissue samples were relatively uniform in immune-related events, such as T

205 cell costimulation, peptide antigen binding, leukocyte migration (Table 1) and allograft rejection,

206 antigen processing and presentation, and graft-versus-host disease (Table 2). Genes in other 
207 modules did not show the same uniform enrichment results in immune-related or any other

208 specific aspect.

209 The expression of immune-related genes and immune-oncology targets

210 According to the Cancer Inflammation \& Immunity Crosstalk PCR Array profile from Qiagen

211 (http://www.sabiosciences.com/rt_pcr_product/HTML/PAHS-181Z.html), a total of $85 \mathrm{key}$

212 genes (Table S2), including 16 immunosuppressive factors, were enrolled for subsequent

213 analysis. The Venn diagram (Fig. 3A) shows the intersection between immune-related genes and

214 DEGs from the GSE40057 and GSE1561 datasets. The Venn diagram (Fig. 3B) shows the

215 intersection between 29 clinical IO targets (Table S2) (Szekely et al., 2018) and DEGs from

216 GSE40057 and GSE1561. There were 15 immune-related genes in GSE40057 (Table S3),

217 including 4 immunosuppressive factors, CD274 (PDL1), CSF2, IL8 (CXCL8) and TGFB1; 31

218 immune-related genes in GSE1561 (Table S3), including 6 immunosuppressive factors,

219 CXCL12, CXCL5, IDO1, MIF, PTGS2 and VEGFA; and 6 immune-related genes in both; 2 IO

220 targets in GSE40057, CD274 and IL8; and 2 IO targets in GSE1561, CXCL12 and IDOI.

221 Interestingly, compared with the luminal-like cell lines and tissue samples, most immune-related

222 genes identified in the basal-like malignancies were upregulated, except CXCL12 (Fig. 3C and

223 3D).

224 GOBO analysis for the immunosuppressive factors screened from cell lines

$225 \mathrm{GOBO}$ analysis showed that $C S F 2, I L 8$ and $T G F \beta 1$ expression levels were inconsistent across

226 the cell lines; there was no information in GOBO for CD274. IL8 had higher average expression

227 levels in basal-like cell lines ( $<00.01$ ) (Fig. 4A); TGF 1 had higher average expression levels in

228 basal-like $(\mathrm{p}<0.01)$ (Fig. 4A) and triple-negative cell lines $(\mathrm{p}<0.01)$ (Fig. 4B). The mRNA levels

229 of CSF2, IL8 and TGFB1 across the 51 breast cancer cell lines are also shown (Fig. 4C).

230 UALCAN and Kaplan-Meier survival analyses for the immunosuppressive factors

231 identified from the screened tissue

232 The UALCAN analysis showed that only CXCL12 (Fig. 5B) had a lower expression level in

233 basal-like (triple-negative) breast cancer compared with luminal-like breast cancer, while CXCL5

234 (Fig. 5A), IDOI (Fig. 5C), MIF (Fig. 5D), PTGS2 (Fig. 5E) and VEGFA (Fig. 5F) all had

235 increased expression levels in basal-like breast cancer.

236 The analysis of overall survival showed that higher CXCL12 (Fig. 6B), IDOI (Fig. 6C) and

$237 P T G S 2$ (Fig. 6E) mRNA expression levels correlated with a comparatively higher survival rate

238 ( $<<0.05$ ), while higher MIF and VEGFA (Fig. 6D, F) expression levels correlated with a lower

239 survival rate $(\mathrm{p}<0.01)$. A further analysis of the screened immunosuppressive factors showed that

240 the combination of CXCL12, IDO1 and PTGS2 was correlated with an increased survival rate

241 (weight: 1:1:1; $<<0.01$; Fig. 6G), while the combination of $M I F$ and $V E G F A$ was correlated with

242 a reduced survival rate (Weight: $1: 1 ; \mathrm{p}<0.01$; Fig. $6 \mathrm{H}$ ). 


\section{Expression of MIF and VEGFA in breast tissue microarrays}

244 To validate whether MIF and VEGFA protein expression is associated with breast cancer

245 malignancy, immunohistochemical detection was performed in a tissue microarray with 90

246 primary tumor tissues and 10 normal breast tissue samples $(p<0.01$; Fig. 7). The results showed

247 that MIF expression was increased dramatically in the metastasis group $(\mathrm{p}<0.05)$ and VEGFA

248 expression positively correlated with tumor grade $(\mathrm{p}<0.05$; Table 3$)$.

\section{CD274 (PD-L1) and IL8 are highly expressed in basal-like breast cancer cell lines}

250

251

252

253

254

255

256

257

258

259

260

261

262

263

264

265

266

267

268

269

270

271

272

273

274

275

276

277

278

279

To validate the expression pattern of the two IO targets, CD274 and IL8, qRT-PCR and western blotting were used to detect the expression of mRNA and protein from 2 basal-like (BT549 and MDA-MB-231) and 2 luminal-like (MCF7 and T47D) breast cancer cell lines (Neve et al., 2006). The qRT-PCR results (Fig. 8A) showed that $C D 274$ and $I L 8$ were upregulated in the basal-like breast cancer cell lines BT549 and MDA-MB-231 ( $<<0.01)$. Similar to the qRT-PCR results, the western blot analysis (Fig. 8B) indicated that CD274 and IL8 protein levels were increased in the BT549 and MDA-MB-231 cell lines compared to those in the MCF7 and T47D cell lines.

\section{Discussion}

The breast tumor microenvironment consists of epithelial tumor cells and extracellular matrix (ECM), including stromal cells such as fibroblasts, adipocytes, endothelial cells and resident immune cells; a multitude of soluble factors; and more recently identified regulatory mediators, such as microRNAs, metabolites and exosomes (Dittmer \& Leyh, 2015). Although cancer progression has been associated with genetic mutations and epigenetic changes in tumor cells, increasing evidence suggests that it is not entirely driven by cancer cell processes and may be influenced by the interplay between cancer cells and their surrounding microenvironment (i.e., tumor-stroma crosstalk) (Criscitiello, Esposito, \& Curigliano, 2014). There is evidence demonstrating that both the stroma and tumor cells evolve upon tumor initiation and progression, which makes the tumor-stroma environment distinct from that of healthy tissue (Quail \& Joyce, 2013). Upon its conversion from normal stroma, tumor stroma thwarts anticancer activities and promotes cancer progression (Granot \& Fridlender, 2015). Therefore, the molecular changes in tumor cells often do not reflect all the changes that occur during tumor-stroma crosstalk in the microenvironment (Morandi \& Chiarugi, 2014).

In this paper, 2 original datasets, GSE40057 and GSE1561, were downloaded from the GEO database. To avoid the inaccuracy produced by 'edged' samples, only 8 cell lines from GSE40057 and 32 tissue samples from GSE1561, representing basal-like and luminal-like groups, were chosen for subsequent analyses due to their great difference in malignancy. In the WGCNA, all genes of the microarray were assigned to corresponding modules based on the weighted gene coexpression network, and the correlations of each module with luminal and basal traits were calculated. Genes involved in the same module were found to be highly 
280

281

282

283

284

285

286

287

288

289

290

291

292

293

294

295

296

297

298

299

300

301

302

303

304

305

306

307

308

309

310

311

312

313

314

315

316

317

318

interconnected and relate to a specific trait, which is different from DEGs that only showed differences in expression levels between groups. Therefore, we chose the WGCNA instead of conventional differential gene expression analysis to screen biological characteristics related to the malignant phenotype of breast cancer. GO and KEGG pathway enrichment analyses for each module showed that genes in the black module of the tissue samples were uniformly enriched in immune-related events, and this module showed a closer correlation with basal-like rather luminal-like breast cancer, indicating that there are certain immune-related events that may play pivotal roles in the malignant phenotype of basal-like breast cancer. However, the enrichment results of modules in the cell lines were not clearly related to immune-related events. This may be because of the tumor-stroma crosstalk in vivo, which is not represented in the cell lines. Therefore, in the field of tumor immune research, the selection of model cell lines or tissue samples may lead to different results, and both have their own unique advantages in scientific research. For instance, the immunosuppressive factors CD274 and IL8 identified from the cell lines were also validated by UALCAN and Kaplan-Meier Plotter, which contained data from tumor tissue rather than the cell lines. This result shows that CD274 and IL8 are also highly expressed in basal-like tumors; increased IL8 expression is associated with poor prognosis, while opposing associations are observed for $C D 274$ (Figure S1). The result of the $C D 274$ analysis did not meet the expectation that higher CD274 expression is associated with poor prognosis. This may be because the tumor tissues contain a variety of stromal cells, such as fibroblasts, adipocytes, endothelial cells and resident immune cells, and CD274 is mainly expressed by antigen-presenting cells (APCs), which need to interact with their receptors in the surrounding stromal cells to exert their effects (Freeman et al., 2000). Therefore, when we use these databases to validate the results from cell lines, which are tumor cells, there may be some inconsistencies. This is also the reason why we analyzed and validated cell lines (pure) and tumor tissues (mixed), separately.

Tumor cells have many strategies for avoiding immune attack, including decreased tumor antigen expression by HLA molecules on the tumor cell surface, downregulation of tumor antigen presentation by dendritic cells, the release of immunosuppressive factors and the activation of regulatory T cells (Raposo, Beirão, Pang, Queiroga, \& Argyle, 2015). Among the many evasion strategies, the release of immunosuppressive factors to induce immunosuppression is an important mechanism for tumor cell evasion of immune surveillance (Jiang \& Shapiro, 2014). Immunosuppression is a reduction of the activation or efficacy of the immune system (Schlößer et al., 2014). Both tumor cells and stromal cells can be induced to synthesize and/or secrete immunosuppressive factors to evade immune surveillance, contributing to tumor initiation and progression (Grivennikov, Greten, \& Karin, 2010). To date, more than 20 immunosuppressive factors produced by tumor and/or stromal cells have been discovered, including transforming growth factor- $\beta 1$ (TGF $\beta 1)(Y$. Wang, Wang, Wang, Zhang, \& Jiang, 2015), prostaglandin E2 (PGE2) (Kalinski, 2012), vascular endothelial growth factor (VEGF) (Shibuya, 2013), interleukin-10 (IL-10) (Geginat et al., 2016), interleukin-4 (IL-4) (Egawa et al., 
319 2013), cyclooxygenase-2 (COX-2) (H. Li et al., 2013), programmed cell death 1 (PDCD1) 320 (Gatalica et al., 2014) and cytotoxic T-lymphocyte associated antigen 4 (CTLA4) (Lan et al., 321 2013). Although therapeutic agents that target immunosuppressive factors, such as BMS-936559, 322 pidilizumab and ipilimumab, have achieved breakthrough responses in cancer immunotherapy 323 and represent one of the most promising strategies for tumor treatment (Schlößer et al., 2014), 324 other immunosuppressive targets are still under investigation, as their roles in tumor malignancy 325 are not completely understood.

326 The analysis of cell lines showed that CD274, CSF2, IL8 and TGFB1 were upregulated in 327 basal-like cell lines compared with luminal-like cell lines. Furthermore, GOBO analysis 328 confirmed that $I L 8$ and $T G F \beta 1$ had higher average expression levels in basal-like and triplenegative cell lines, indicating that $I L 8$ and $T G F \beta 1$ may be associated with a malignant phenotype. The analysis of tissue samples revealed that CXCL5, IDO1, PTGS2, MIF and VEGFA were upregulated in basal-like tissues compared with luminal-like tissues, which was also confirmed by UALCAN analysis. In addition, the Kaplan-Meier survival analysis showed that increased $M I F$ and $V E G F A$ expression levels in breast cancer patients correlated with a reduced survival rate. These findings suggest that the increased expression levels of $M I F$ and $V E G F A$ contribute to the malignant phenotype of breast cancer. Our IHC results also validated this result.

Cancer-derived IL8 may result in the recruitment and activation of tumor-associated neutrophils (TANs) and myeloid-derived suppressor cells (MDSCs) to contribute to the tumor microenvironment and immune suppression and activate endothelial cells for angiogenesis (Waugh \& Wilson, 2008). IL8 functions by activating the PI3K-Akt and PLC-PKC signaling pathways. These two signaling pathways have been demonstrated to be associated with angiogenesis, cell survival, and migration (Cheng et al., 2008). Overexpressed IL8 is associated with accelerated breast cancer progression, an increased tumor load, and the presence of distant metastasis, ultimately leading to poor survival (Singh, Simoes, Howell, Farnie, \& Clarke, 2013). TGF $\beta 1$ is a well-known family involved in various tumor processes, such as induction of epithelial-mesenchymal transition and the regulation of cancer migration and invasion (J. Xu, Lamouille, \& Derynck, 2009)._The overexpression of MIF has been demonstrated in the progression of multiple cancers, such as ovarian cancer (Krockenberger et al., 2012), hepatocellular carcinoma (D. Wang et al., 2014), gastric cancer (He et al., 2006) and other malignant cancers. Several studies have elucidated the multiple roles of MIF in the breast cancer microenvironment, including increasing the recruitment of immunosuppressive cells (Simpson, Templeton, \& Cross, 2012), inducing angiogenesis and breast cancer cell trans-endothelial migration (Martinez et al., 2014). In addition, MIF also acts on tumor cells to facilitate cell proliferation and cell survival (Lue et al., 2007). Thus far, the VEGFA protein has been identified as a major factor that contributes to tumor angiogenesis and malignant progression in a variety of cancers (Q. Li et al., 2017; Yang, Xiong, Zuo, Liu, \& Zhang, 2018). In recent years, a number of antiangiogenic drugs have been designed and showed significant effects in chemotherapy. Unfortunately, some of these drugs (e.g., bevacizumab) showed limited effects in specific breast cancer conditions (Bergh et al., 2012; Varinska, Gal, Mojzisova, Mirossay, \& 
359 Mojzis, 2015). Therefore, patients need to be evaluated before the implementation of

360 antiangiogenic therapy. Prospectively, VEGFA may act as an evaluation factor to identify breast

361 cancer patients who might benefit from antiangiogenic therapy.

362 Currently, a variety of IO targets are available in clinical therapy (Szekely et al., 2018).

363 Among the genes identified in our results, CD274, IL8, CXCL12 and IDOI (Fig. 3B) are

364 included in these IO targets. Since the Kaplan-Meier survival analysis showed that CXCL12 and

365 IDO1 were not associated with the malignant phenotype of breast cancer, they were not studied

366 further. The high expression levels of CD274 and IL8 in basal-like breast cancer were validated

367 by qRT-PCR and western blot, suggesting that clinical CD274- and $I L 8$-targeting therapies may

368 be more suitable for basal-like breast cancer. Other immunosuppressive factors in this study are

369 also worth further study in the field of subtype-specific IO targeted therapy for breast cancer.

\section{Conclusions}

371 Using online databases, model reconstruction and comparisons of the mRNA expression

372 profiles of luminal-like and basal-like cell lines and primary breast cancer tissues, 4

373 immunosuppressive factors associated with a malignant phenotype in breast cancer were

374 identified and validated. Such molecules could be used as biomarkers for malignant breast cancer

375 phenotypes and prognosis. In addition, 2 immunosuppressive factors were confirmed as clinical

376 IO therapeutic targets, which may be most suitable for the treatment of basal-like breast cancer.

377 However, because the majority of immune-related factors have diverse roles in disease pathology

378 and we still lack a complete understanding of the relationship between immunosuppressive

379 factors and breast cancer malignancy, the feasibility of the clinical application of the identified

380 factors as drug targets and prognosis predictors warrants further investigation. 
381 Acknowledgments: The authors would like to thank Ping Zhang, Qingmei Zhong, Xianhe Yang,

382 Wu Wang, Di Yao and Yingqun Xiao at Department of Pathology, Affiliated Infectious Diseases

383 Hospital, Nanchang University, for their technical assistance.

384

385

\section{References}

386

Bahnassy, A., Mohanad, M., Ismail, M. F., Shaarawy, S., El-Bastawisy, A., \& Zekri, A.-R. N. (2015). Molecular biomarkers for prediction of response to treatment and survival in triple negative breast cancer patients from Egypt. Experimental and Molecular Pathology, 99(2), 303311. doi: 10.1016/j.yexmp.2015.07.014

Barrett, T., Wilhite, S. E., Ledoux, P., Evangelista, C., Kim, I. F., Tomashevsky, M., . . . Soboleva, A. (2012). NCBI GEO: archive for functional genomics data sets--update. Nucleic Acids Res, 41(D1), D991-D995. doi: 10.1093/nar/gks1193

393 Bergh, J., Mariani, G., Cardoso, F., Liljegren, A., Awada, A., Vigano, L., . . Gianni, L. (2012). Clinical and pharmacokinetic study of sunitinib and docetaxel in women with advanced breast cancer. Breast, 21(4), 507-513. doi: 10.1016/j.breast.2012.01.012

396 Cancer facts \$figures 2016. (2016).

397 Chandrashekar, D. S., Bashel, B., Balasubramanya, S. A. H., Creighton, C. J., Ponce-Rodriguez, 398 I., Chakravarthi, B. V., \& Varambally, S. (2017). UALCAN: A Portal for Facilitating Tumor 399 Subgroup Gene Expression and Survival Analyses1. Neoplasia, 19(8), 649-658. doi:

400 10.1016/j.neo.2017.05.002

401 Cheng, G. Z., Park, S., Shu, S., He, L., Kong, W., Zhang, W., . . Cheng, J. Q. (2008). Advances 402 of AKT pathway in human oncogenesis and as a target for anti-cancer drug discovery. Curr 403 Cancer Drug Targets, 8(1), 2-6.

404 Criscitiello, C., Esposito, A., \& Curigliano, G. (2014). Tumor-stroma crosstalk. Current Opinion 405 in Oncology, 26(6), 551-555. doi: 10.1097/cco.0000000000000122

406 Dittmer, J., \& Leyh, B. (2015). The impact of tumor stroma on drug response in breast cancer. 407 Semin Cancer Biol, 31, 3-15. doi: 10.1016/j.semcancer.2014.05.006

408 Egawa, M., Mukai, K., Yoshikawa, S., Iki, M., Mukaida, N., Kawano, Y., . . Karasuyama, H. 409 (2013). Inflammatory monocytes recruited to allergic skin acquire an anti-inflammatory M2 410 phenotype via basophil-derived interleukin-4. Immunity, 38(3), 570-580.

411 Farmer, P., Bonnefoi, H., Becette, V., Tubiana-Hulin, M., Fumoleau, P., Larsimont, D., ... Iggo, 412 R. (2005). Identification of molecular apocrine breast tumours by microarray analysis. Oncogene, 413 24(29), 4660-4671. doi: 10.1038/sj.onc.1208561 
414 Freeman, G. J., Long, A. J., Iwai, Y., Bourque, K., Chernova, T., Nishimura, H., . . Honjo, T. 415 (2000). Engagement of the PD-1 immunoinhibitory receptor by a novel B7 family member leads 416 to negative regulation of lymphocyte activation. J Exp Med, 192(7), 1027-1034.

417 Gandellini, P., Andriani, F., Merlino, G., D'Aiuto, F., Roz, L., \& Callari, M. (2015). Complexity 418 in the tumour microenvironment: Cancer associated fibroblast gene expression patterns identify 419 both common and unique features of tumour-stroma crosstalk across cancer types. Semin Cancer 420 Biol, 35, 96-106. doi: 10.1016/j.semcancer.2015.08.008

421 Gatalica, Z., Snyder, C., Maney, T., Ghazalpour, A., Holterman, D. A., Xiao, N., .. . Vranic, S. 422 (2014). Programmed cell death 1 (PD-1) and its ligand (PD-L1) in common cancers and their 423 correlation with molecular cancer type. Cancer Epidemiology Biomarkers \& Prevention, 23(12), 424 2965-2970.

425 Geginat, J., Larghi, P., Paroni, M., Nizzoli, G., Penatti, A., Pagani, M., . . Flavell, R. A. (2016). 426 The light and the dark sides of Interleukin-10 in immune-mediated diseases and cancer. Cytokine 427 \& Growth Factor Reviews.

428 Gentleman, R. C., Carey, V. J., Bates, D. M., Bolstad, B., Dettling, M., Dudoit, S., . . Zhang, J. 429 (2004). Bioconductor: open software development for computational biology and bioinformatics. 430 Genome biology, 5(10), R80. doi: 10.1186/gb-2004-5-10-r80

431 Granot, Z., \& Fridlender, Z. G. (2015). Plasticity beyond Cancer Cells and the 432 "Immunosuppressive Switch". Cancer Research, 75(21), 4441-4445. doi: 10.1158/0008433 5472.can-15-1502

434 Grivennikov, S. I., Greten, F. R., \& Karin, M. (2010). Immunity, inflammation, and cancer. Cell, 435 140(6), 883-899. doi: 10.1016/j.cell.2010.01.025

436 Gyorffy, B., Surowiak, P., Budczies, J., \& Lanczky, A. (2013). Online survival analysis software 437 to assess the prognostic value of biomarkers using transcriptomic data in non-small-cell lung 438 cancer. PLoS One, 8(12), e82241. doi: 10.1371/journal.pone.0082241

439 He, X. X., Yang, J., Ding, Y. W., Liu, W., Shen, Q. Y., \& Xia, H. H. (2006). Increased epithelial 440 and serum expression of macrophage migration inhibitory factor (MIF) in gastric cancer:

441 potential role of MIF in gastric carcinogenesis. Gut, 55(6), 797-802. doi:

442 10.1136/gut.2005.078113

443 Huang da, W., Sherman, B. T., \& Lempicki, R. A. (2009). Systematic and integrative analysis of 444 large gene lists using DAVID bioinformatics resources. Nat Protoc, 4(1), 44-57. doi:

445 10.1038/nprot.2008.211

446 Jezequel, P., Campone, M., Gouraud, W., Guerin-Charbonnel, C., Leux, C., Ricolleau, G., \& 447 Campion, L. (2012). bc-GenExMiner: an easy-to-use online platform for gene prognostic 448 analyses in breast cancer. Breast Cancer Res Treat, 131(3), 765-775. doi: 10.1007/s10549-011$449 \quad 1457-7$ 
450 Jiang, X., \& Shapiro, D. J. (2014). The immune system and inflammation in breast cancer. 451 Molecular and Cellular Endocrinology, 382(1), 673-682. doi: 10.1016/j.mce.2013.06.003

452 Kalinski, P. (2012). Regulation of immune responses by prostaglandin E2. The Journal of 453 Immunology, 188(1), 21-28.

454 Krockenberger, M., Kranke, P., Hausler, S., Engel, J. B., Horn, E., Nurnberger, K., . . . Honig, A. 455 (2012). Macrophage migration-inhibitory factor levels in serum of patients with ovarian cancer 456 correlates with poor prognosis. Anticancer Res, 32(12), 5233-5238.

457 Lan, K.-H., Liu, Y.-C., Shih, Y.-S., Tsaid, C.-L., Yen, S.-H., \& Lan, K.-L. (2013). A DNA 458 vaccine against cytotoxic T-lymphocyte associated antigen-4 (CTLA-4) prevents tumor growth. 459 Biochem Biophys Res Commun, 440(2), 222-228.

460 Langfelder, P., \& Horvath, S. (2008). WGCNA: an R package for weighted correlation network 461 analysis. BMC Bioinformatics, 9, 559. doi: 10.1186/1471-2105-9-559

462 Li, H., Edin, M. L., Bradbury, J. A., Graves, J. P., DeGraff, L. M., Gruzdev, A., . . Bortner, C. 463 D. (2013). Cyclooxygenase-2 inhibits T helper cell type 9 differentiation during allergic lung 464 inflammation via down-regulation of IL-17RB. American journal of respiratory and critical care 465 medicine, 187(8), 812-822.

466 Li, Q., Kan, X., Yin, J., Sun, L., Wang, Y., Li, Y., . . Zhu, X. (2017). Chamaejasmine B Induces 467 the Anergy of Vascular Endothelial Cells to VEGFA Pro-angiogenic Signal by Autophagic 468 Regulation of VEGFR2 in Breast Cancer. Front Pharmacol, 8, 963. doi:

469 10.3389/fphar.2017.00963

470 Liu, X., \& Wang, Q. (2015). Screening of feature genes in distinguishing different types of 471 breast cancer using support vector machine. OncoTargets and Therapy, 2311. doi:

$47210.2147 /$ ott.s85271

473 Lue, H., Thiele, M., Franz, J., Dahl, E., Speckgens, S., Leng, L., . . Bernhagen, J. (2007).

474 Macrophage migration inhibitory factor (MIF) promotes cell survival by activation of the Akt 475 pathway and role for CSN5/JAB1 in the control of autocrine MIF activity. Oncogene, 26(35), 476 5046-5059. doi: 10.1038/sj.onc. 1210318

477 Luo, D., Wilson, J. M., Harvel, N., Liu, J., Pei, L., Huang, S., . . Shi, H. (2013). A systematic 478 evaluation of miRNA:mRNA interactions involved in the migration and invasion of breast 479 cancer cells. $J$ Transl Med, 11(1), 57. doi: 10.1186/1479-5876-11-57

480 Martinez, L. M., Vallone, V. B., Labovsky, V., Choi, H., Hofer, E. L., Feldman, L., . . . 481 Chasseing, N. A. (2014). Changes in the peripheral blood and bone marrow from untreated 482 advanced breast cancer patients that are associated with the establishment of bone metastases. 483 Clin Exp Metastasis, 31(2), 213-232. doi: 10.1007/s10585-013-9622-5

484 Morandi, A., \& Chiarugi, P. (2014). Metabolic implication of tumor:stroma crosstalk in breast 485 cancer. Journal of Molecular Medicine, 92(2), 117-126. doi: 10.1007/s00109-014-1124-7 
486 Neve, R. M., Chin, K., Fridlyand, J., Yeh, J., Baehner, F. L., Fevr, T., . . . Gray, J. W. (2006). A 487 collection of breast cancer cell lines for the study of functionally distinct cancer subtypes.

488 Cancer Cell, 10(6), 515-527. doi: 10.1016/j.ccr.2006.10.008

489 Prat, A., Pineda, E., Adamo, B., Galvan, P., Fernandez, A., Gaba, L., . . Munoz, M. (2015).

490 Clinical implications of the intrinsic molecular subtypes of breast cancer. Breast, 24 Suppl 2,

491 S26-35. doi: 10.1016/j.breast.2015.07.008

492 Quail, D. F., \& Joyce, J. A. (2013). Microenvironmental regulation of tumor progression and 493 metastasis. Nature medicine, 19(11), 1423-1437. doi: 10.1038/nm.3394

494 Raposo, T. P., Beirão, B. C. B., Pang, L. Y., Queiroga, F. L., \& Argyle, D. J. (2015).

495 Inflammation and cancer: Till death tears them apart. The Veterinary Journal, 205(2), 161-174.

496 doi: 10.1016/j.tvj1.2015.04.015

497 Ringner, M., Fredlund, E., Hakkinen, J., Borg, A., \& Staaf, J. (2011). GOBO: gene expression498 based outcome for breast cancer online. PLoS One, 6(3), e17911. doi:

499 10.1371/journal.pone.0017911

500 Schlößer, H. A., Theurich, S., Shimabukuro-Vornhagen, A., Holtick, U., Stippel, D. L., \&

501 Bergwelt-Baildon, M. v. (2014). Overcoming tumor-mediated immunosuppression.

502 Immunotherapy, 6(9), 973-988. doi: 10.2217/imt.14.58

503 Shibuya, M. (2013). Vascular endothelial growth factor and its receptor system: physiological 504 functions in angiogenesis and pathological roles in various diseases. Journal of biochemistry, 505 153(1), 13-19.

506 Simpson, K. D., Templeton, D. J., \& Cross, J. V. (2012). Macrophage migration inhibitory factor 507 promotes tumor growth and metastasis by inducing myeloid-derived suppressor cells in the 508 tumor microenvironment. J Immunol, 189(12), 5533-5540. doi: 10.4049/jimmunol.1201161

509 Singh, J. K., Simoes, B. M., Howell, S. J., Farnie, G., \& Clarke, R. B. (2013). Recent advances 510 reveal IL-8 signaling as a potential key to targeting breast cancer stem cells. Breast Cancer Res, 511 15(4), 210. doi: 10.1186/bcr3436

512 Szekely, B., Bossuyt, V., Li, X., Wali, V. B., Patwardhan, G. A., Frederick, C., . . Pusztai, L.

513 (2018). Immunological differences between primary and metastatic breast cancer. Ann Oncol.

514 doi: 10.1093/annonc/mdy399

515 Tomao, S., Tomao, F., Rossi, L., Zaccarelli, E., Caruso, D., Minozzi, M., . . Vici. (2015).

516 Triple-negative breast cancer: new perspectives for targeted therapies. OncoTargets and Therapy,

517 177. doi: 10.2147/ott.s67673

518 Varinska, L., Gal, P., Mojzisova, G., Mirossay, L., \& Mojzis, J. (2015). Soy and breast cancer:

519 focus on angiogenesis. Int J Mol Sci, 16(5), 11728-11749. doi: 10.3390/ijms160511728

520 Wang, D., Luo, L., Chen, W., Chen, L. Z., Zeng, W. T., Li, W., \& Huang, X. H. (2014).

521 Significance of the vascular endothelial growth factor and the macrophage migration inhibitory

Peer] reviewing PDF | (2019:03:35676:1:1:CHECK 15 May 2019) 
522 factor in the progression of hepatocellular carcinoma. Oncol Rep, 31(3), 1199-1204. doi:

523 10.3892/or.2013.2946

524 Wang, Y., Wang, X., Wang, X., Zhang, D., \& Jiang, S. (2015). Effect of transforming growth

525 factor-beta1 869C/T polymorphism and radiation pneumonitis. Int J Clin Exp Pathol, 8(3), 2835-

5262839.

527 Waugh, D. J., \& Wilson, C. (2008). The interleukin-8 pathway in cancer. Clin Cancer Res,

528 14(21), 6735-6741. doi: 10.1158/1078-0432.ccr-07-4843

529 Xu, J., Lamouille, S., \& Derynck, R. (2009). TGF-beta-induced epithelial to mesenchymal

530 transition. Cell Res, 19(2), 156-172. doi: 10.1038/cr.2009.5

531 Xu, M., Du, X., Liu, M., Li, S., Li, X., Fu, Y.-X., \& Wang, S. (2012). The tumor

532 immunosuppressive microenvironment impairs the therapy of anti-HER2/neu antibody. Protein

533 Cell, 3(6), 441-449. doi: 10.1007/s13238-012-2044-3

534 Yang, P., Xiong, J., Zuo, L., Liu, K., \& Zhang, H. (2018). miR1405p regulates cell migration

535 and invasion of nonsmall cell lung cancer cells through targeting VEGFA. Mol Med Rep. doi:

$536 \quad 10.3892 / \mathrm{mmr} .2018 .9291$

537 


\section{Figure 1}

Unsupervised analysis.

(A) Principal components of all genes. The first two PCAs are plotted. The three major groups are colored blue (basal-like), red (luminal-like) and green (edged samples). (B) Hierarchical clustering of all samples. According to theproximities between the samples, 8 cell lines (4 basal-like and 4 luminal-like) from GSE40057, and 32 tissue samples (16 basal-like and 16 luminal-like) from GSE1561 were chosen for subsequent analyses. 
(A)

GSE40057

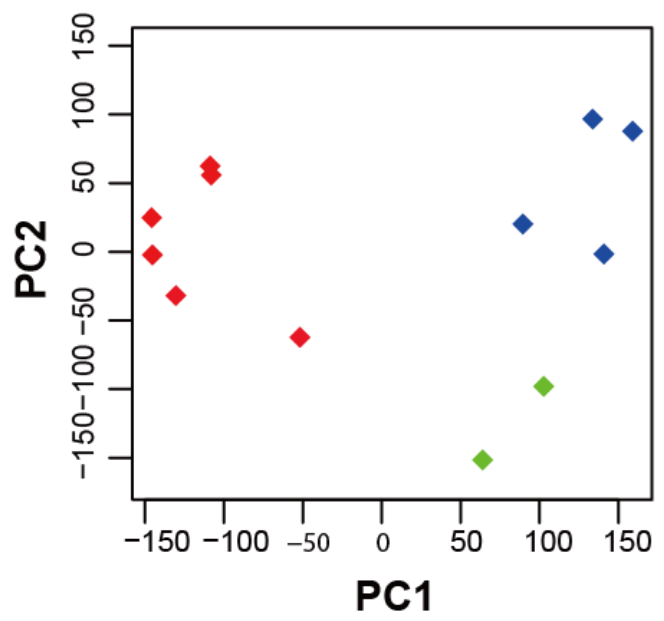

GSE1561

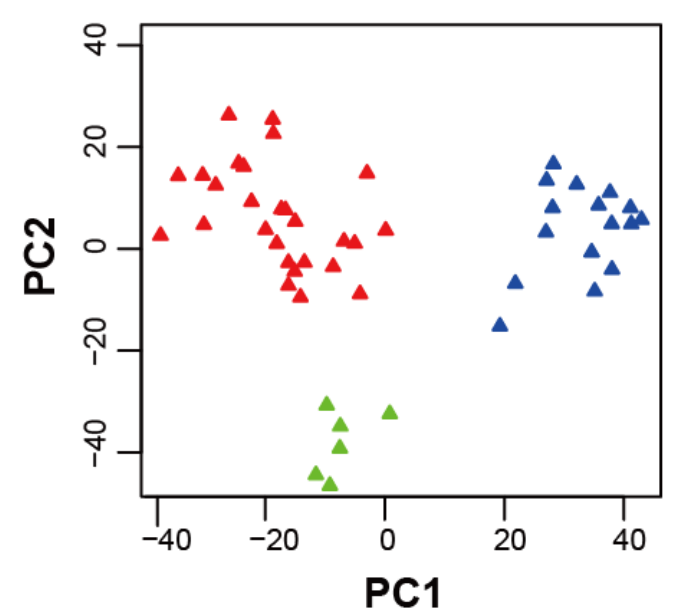

(B)

\section{GSE40057}

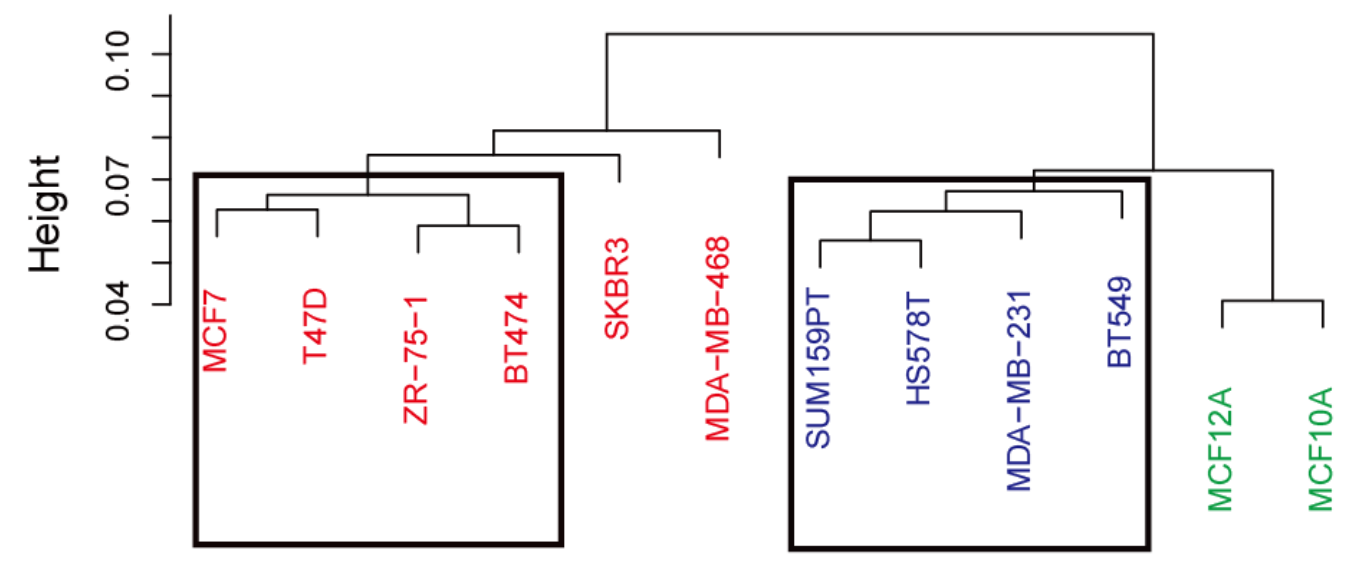

\section{GSE1561}

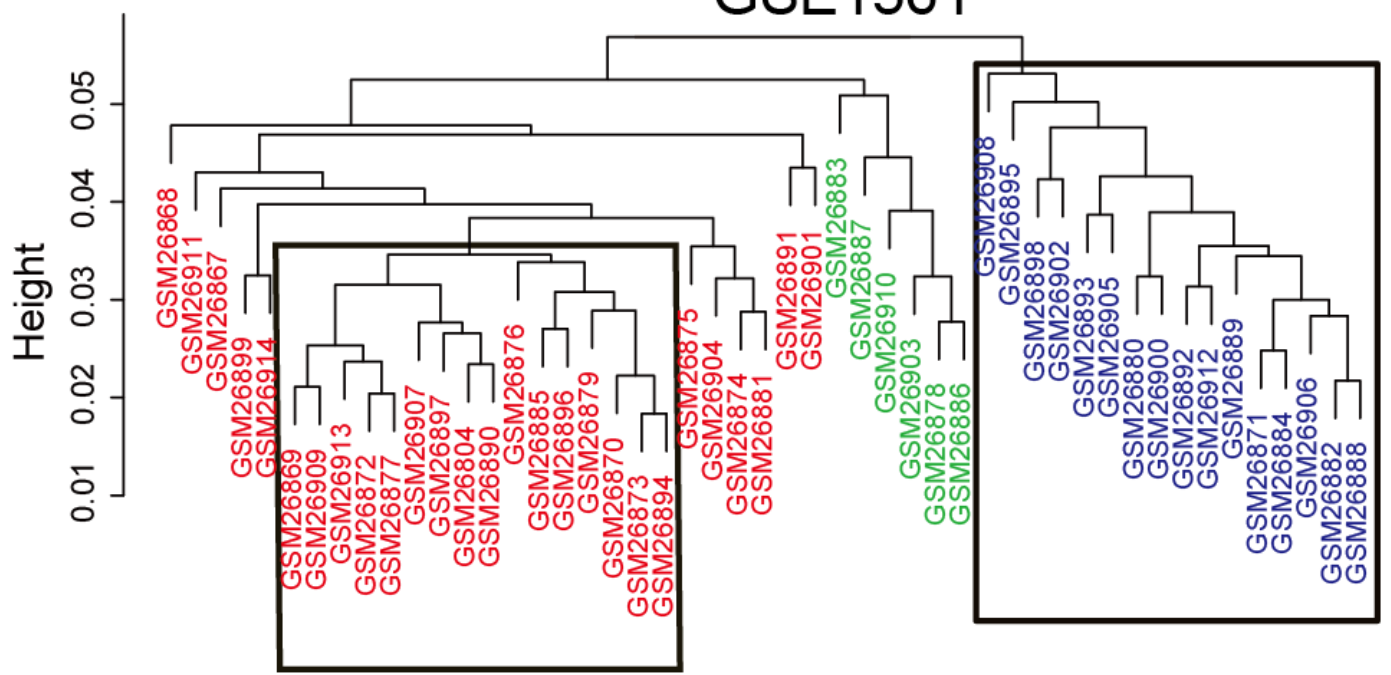




\section{Figure 2}

WGCNA of GSE40057 (A, B) and GSE1561 (C, D).

(A) A total of 20283 genes were assigned to 25 modules. The gene dendrogram is shown in the top portion, and the 25 gene modules are shown in the bottom portion. (B, D) The relationship between modules and traits. The upper score in each box represents the module significance (MS) score, and the lower value represents the corresponding $p$-value. If a box is red ( $M S>0$ ), the module in which the box is located is thought to be correlated with the trait; the lower the p-value, the higher the MS score is, and the redder the color of the box, the stronger the correlation is. (C) A total of 12752 genes were assigned to 6 modules.

(A)

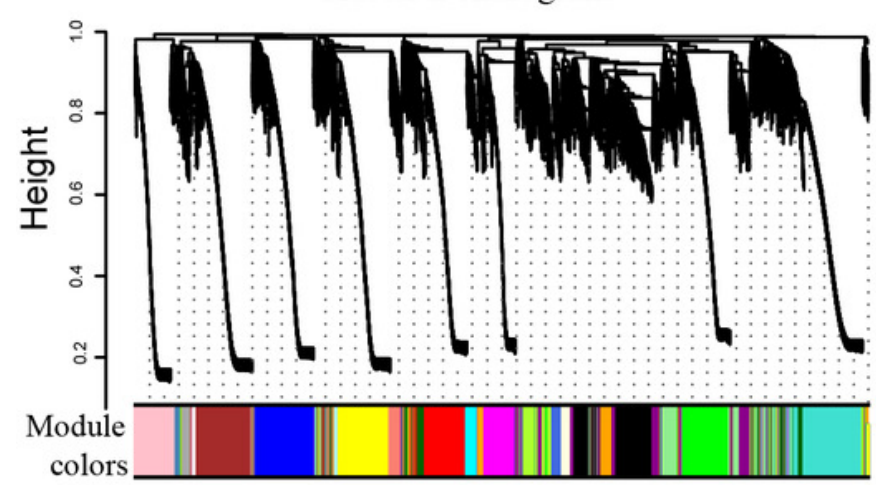

(C)

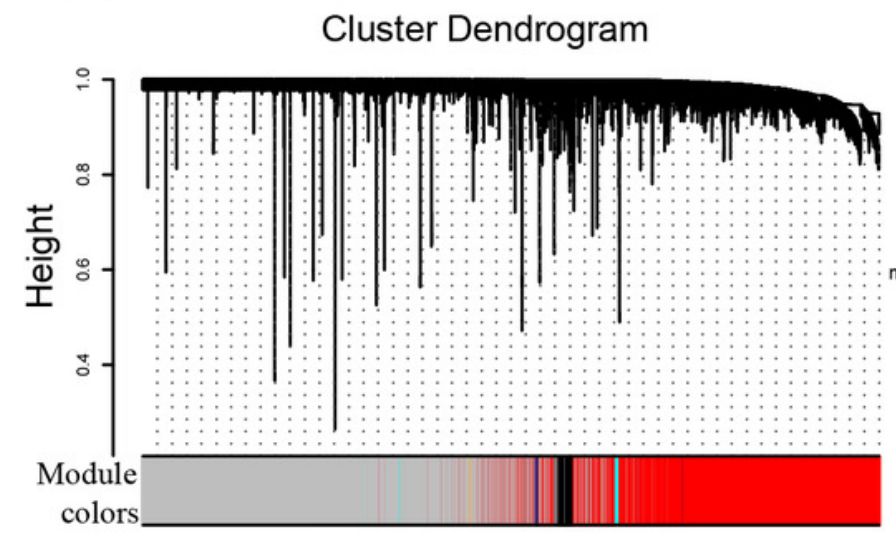

(B)

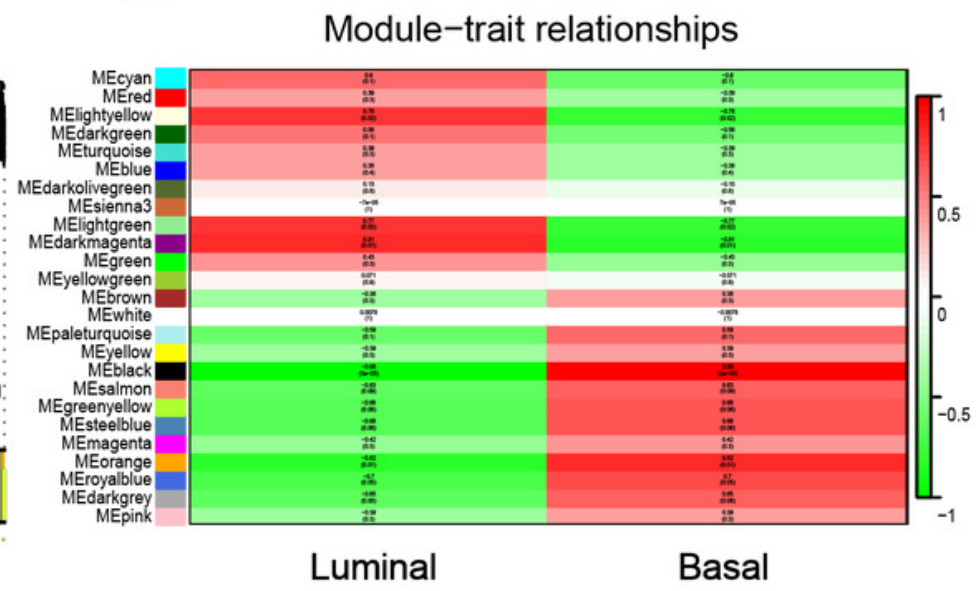

(D)

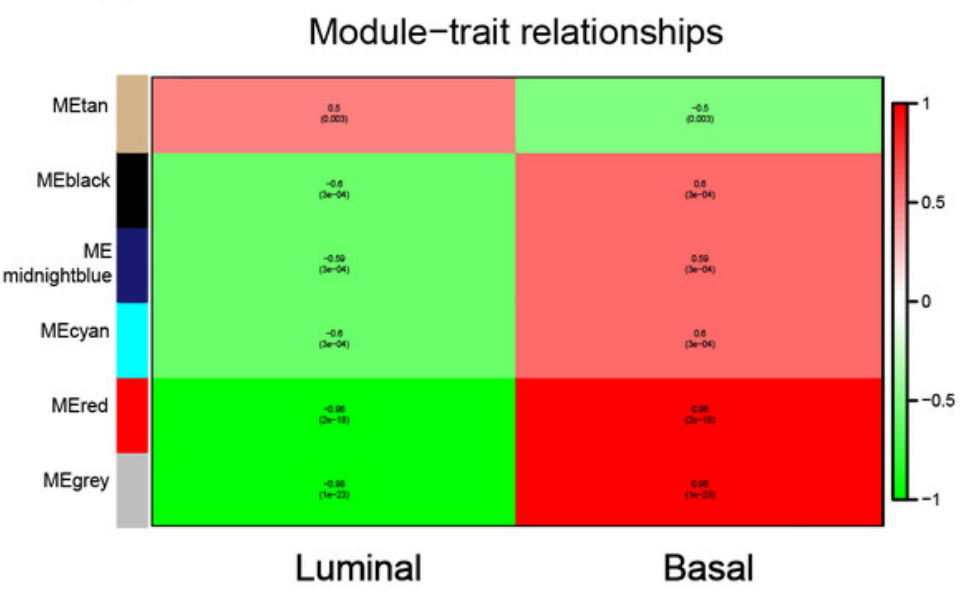




\section{Figure 3}

The expression of immune-related genes in cell lines and tissue samples.

(A) The Venn diagram shows the intersection between immune-related genes and DEGs from GSE40057 and GSE1561. (B) The Venn diagram shows the intersection between 29 clinical IO therapeutic targets and DEGs from GSE40057 and GSE1561: CD274and IL8 from GSE40047; CXCL12 and IDO1 from GSE1561. (C, D) Hierarchical clustering analysis of immune-related gene expression in cell lines (C) and tissue samples (D). Each row corresponds to an Immunerelated gene, and each column corresponds to an independent cell or tissue sample. The darker color indicates increased expression, and the immune-related genes in red letters are thought to be immunosuppressive factors. $*=10$ target. 
(A)

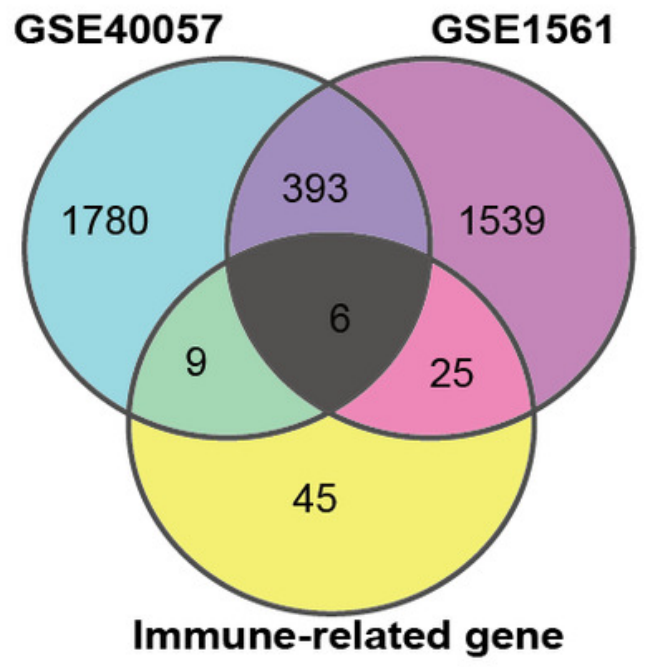

(B)

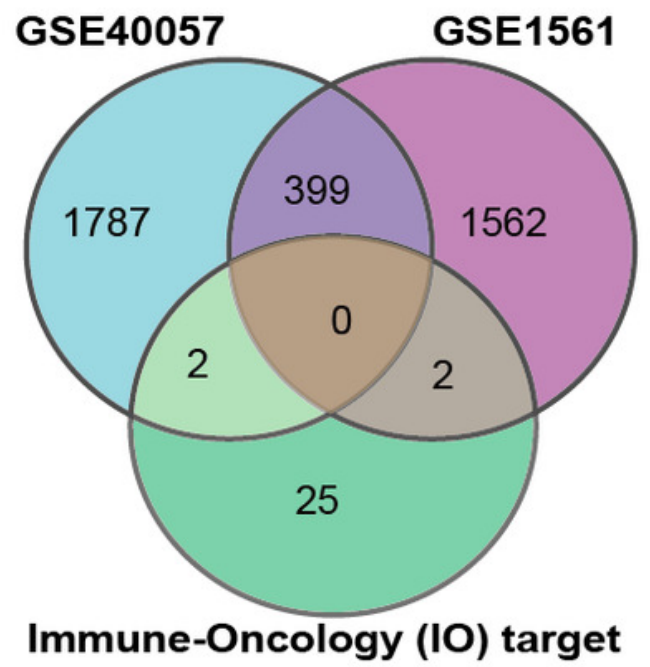

(C)

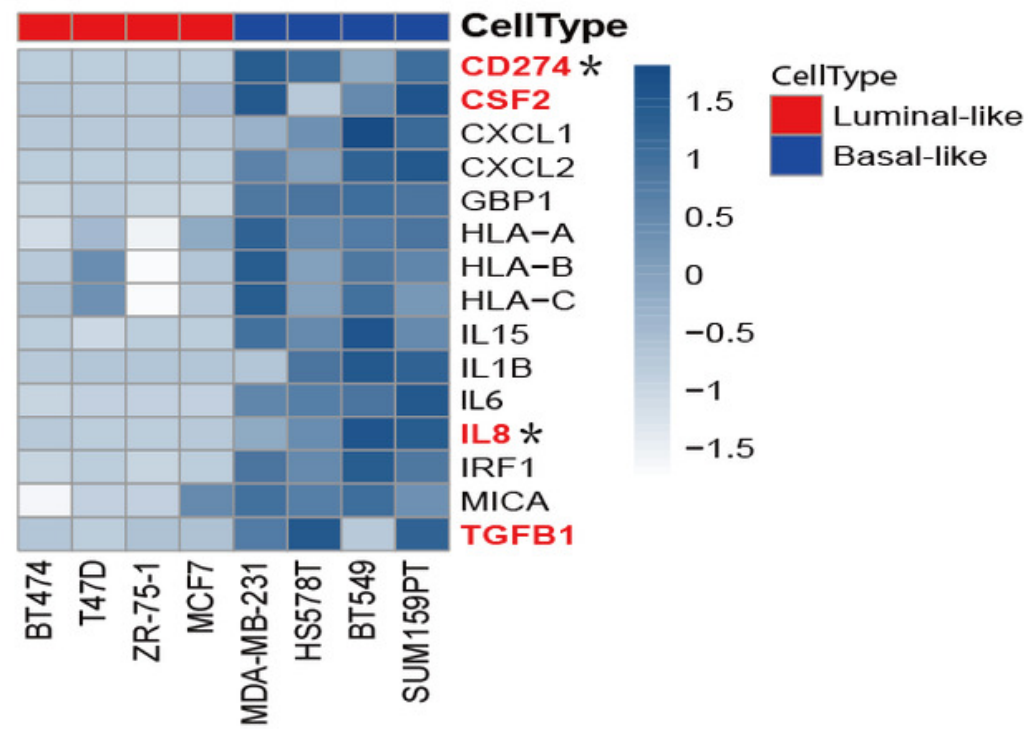

(D)

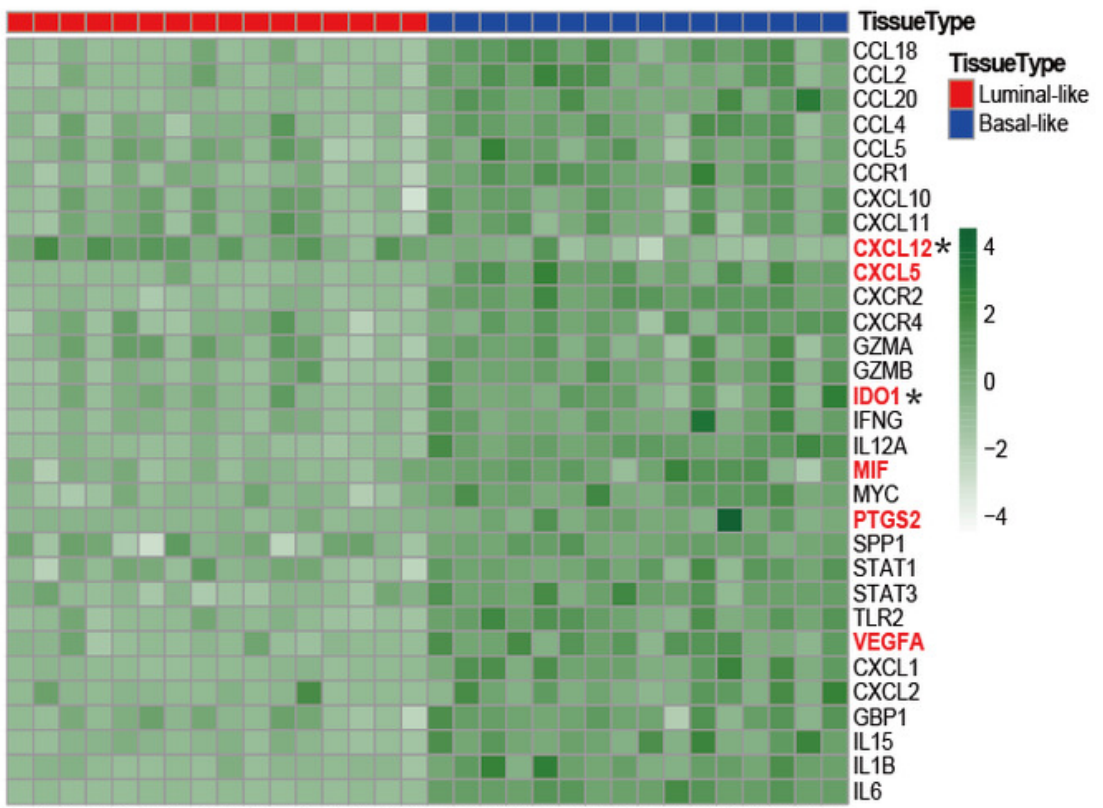




\section{Figure 4}

CSF2, IL8 and TGF $\beta 1$ expression in human breast cancer cell lines with GOBO analysis.

(A) Box plots of CSF2, IL8 and TGF $\beta 1$ expression across 51 breast cancer cell lines grouped into basal A (red), basal B (gray) and luminal (blue) subgroups. The expression levels of IL8 and TGF $\beta 1$ in the basal $A$ and $B$ subgroups were higher than those in the luminal subgroup $(p<0.01$ ), while the CSF2 results were not statistically significant. (B) Box plots of CSF2, IL8 and TGF $\beta 1$ expression across 51 breast cancer cell lines grouped into triple-negative (TN), HER2-positive and hormone receptor-positive (HR) groups. The expression level of TGF $\beta 1$ in the TN subgroup was higher than that in the HER2 and HR subgroups $(p<0.01)$, while the CSF2 and IL8 results were not statistically significant. (C) CSF2,IL8 and TGF $\beta 1$ mRNA levels across 51 breast cancer cell lines. 
(A)

CSF2 $p=0.29755$

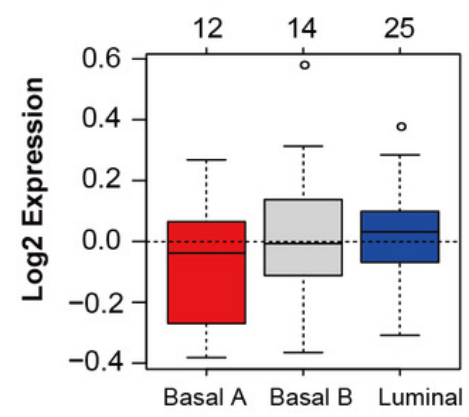

(B)

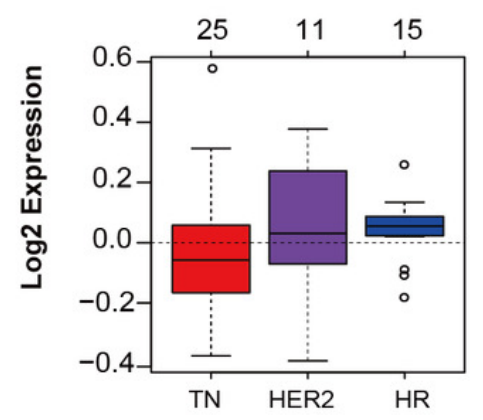

IL8 $p=0.00058$

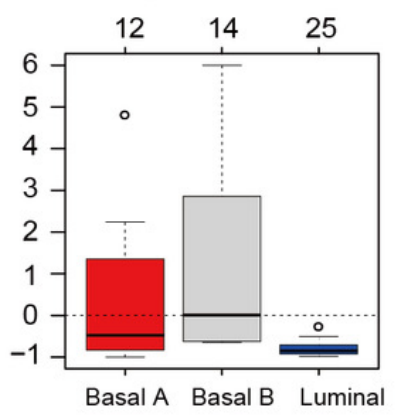

IL8 $p=0.05806$

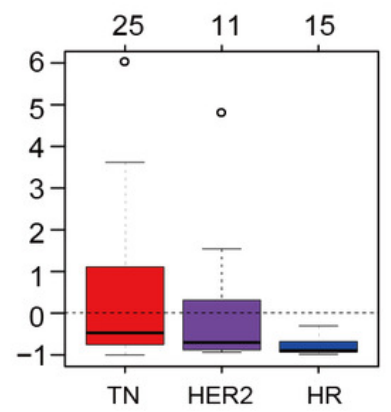

TGFB1 $\mathrm{p}=<0.00001$

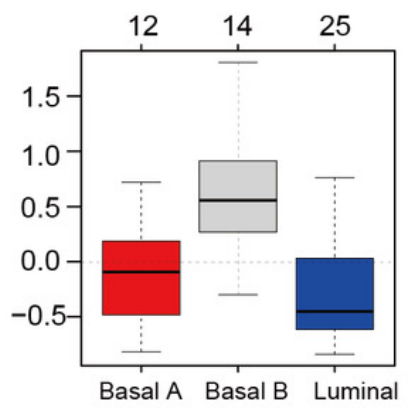

TGFB1 $p=\mathbf{0 . 0 0 8 2 7}$

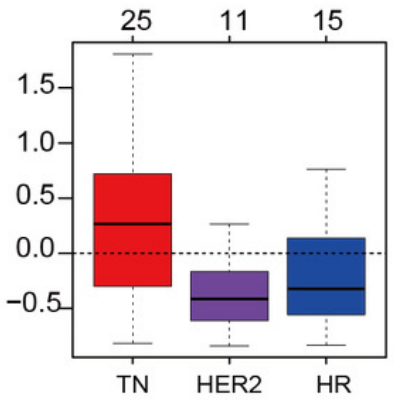

(C)

Neve expression for each cell line: CSF2

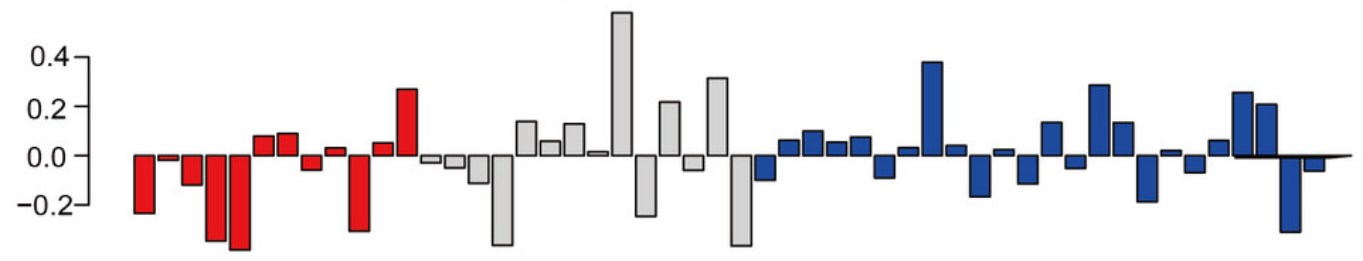

Neve expression for each cell line: IL8
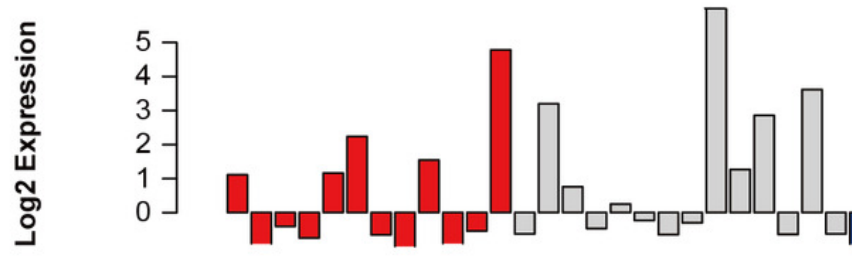

Neve expression for each cell line: TGFB1

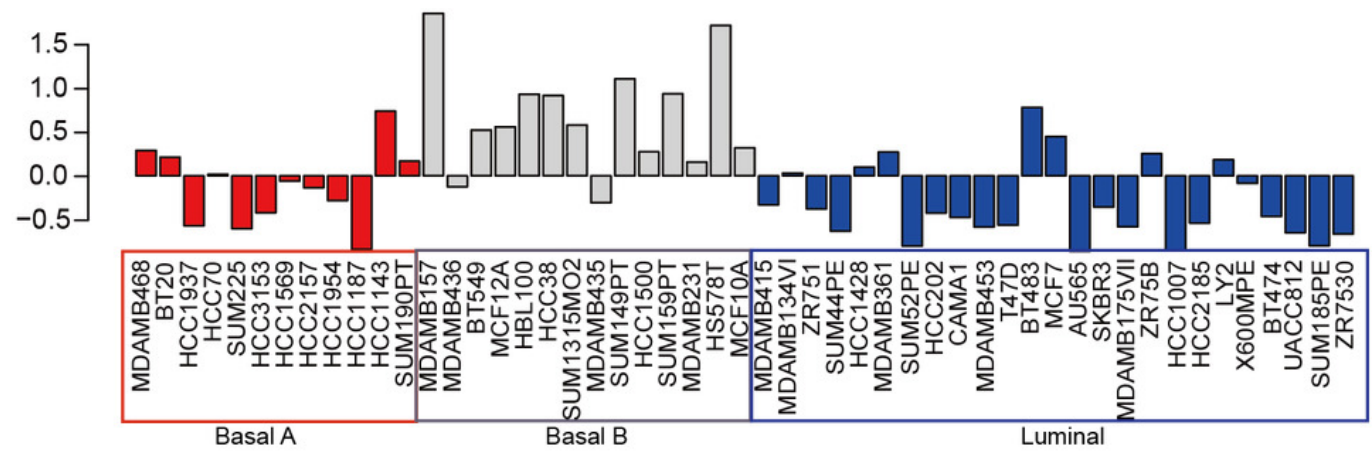

PeerJ reviewing PDF | (2019:03:35676:1:1:CHECK 15 May 2019) 
Figure 5

Expression of the six immuosuppressive factors in breast cancer based on breast cancer subtypes.

( $A, C, D, E$, and F) CXCL5, IDO1, MIF, PTGS2 and VEGFA are expressed at higher levels in basal (triple-negative) breast cancer compared with luminal breast cancer. (B) CXCL12 is expressed at lower levels in basal (triple-negative) breast cancer. ${ }^{*} p<0.05, * * p<0.005$, and $* * * p<0.001$.
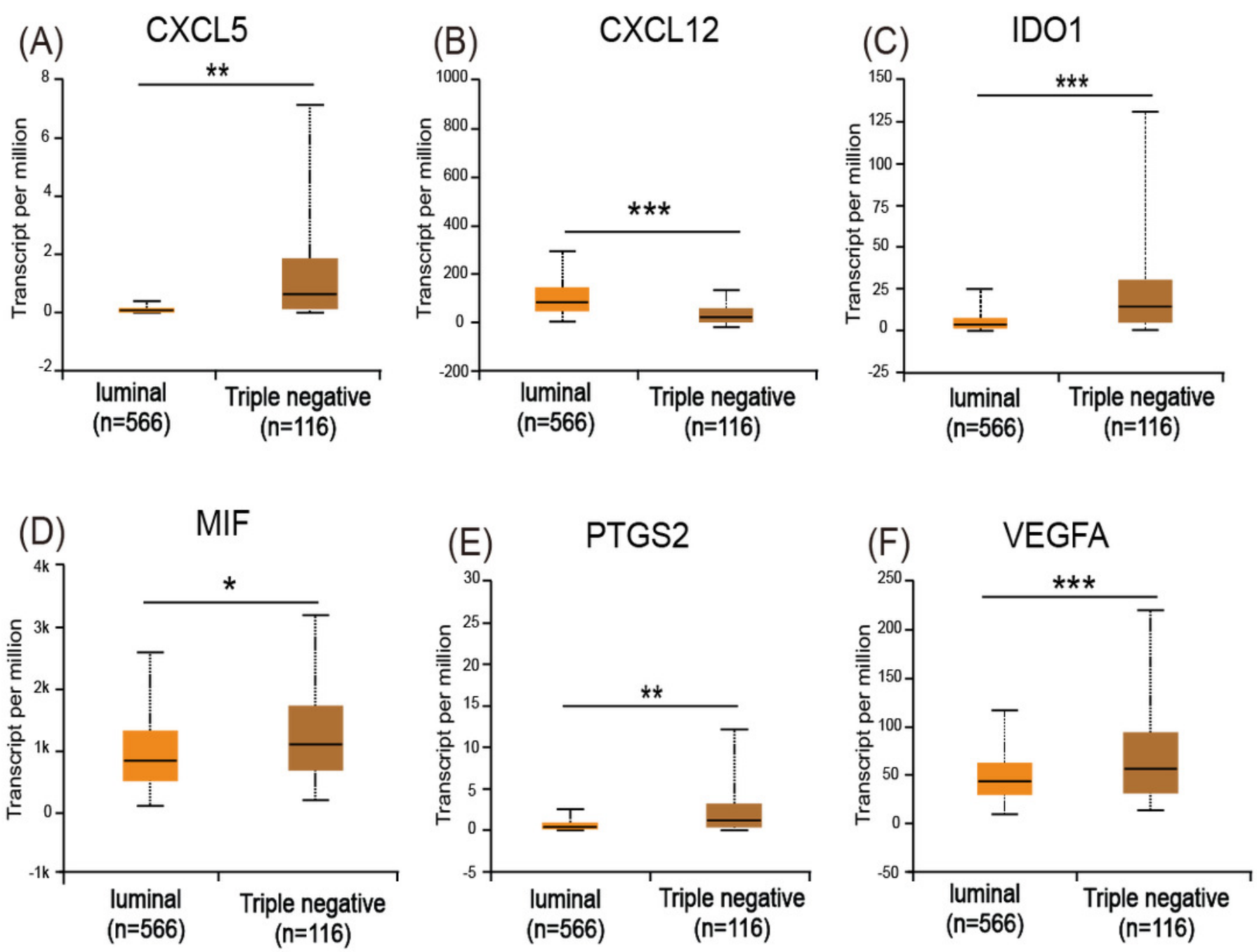


\section{Figure 6}

Kaplan-Meier Plotter determined the relationship between survival rate and mRNA expression levels of 6 immunosuppressive factors using microarray data of 3951 patients.

(B-F) Increased CXCL12, IDO1 and PTGS2 mRNA expression levels correlated with a comparatively increased survival rate $(p<0.05)$, while increased MIF and VEGFA expression levels correlated with a reduced survival rate $(p<0.01)$. $(G, H)$ The combination of CXCL12, IDO1 and PTGS2 correlated with an increased survival rate (weight: 1:1:1) $(p<0.01)$, while the combination of MIF and VEGFA correlated with a reduced survival rate (weight: 1:1) $(p<0.01) . H R=$ hazard ratio. 
(A)

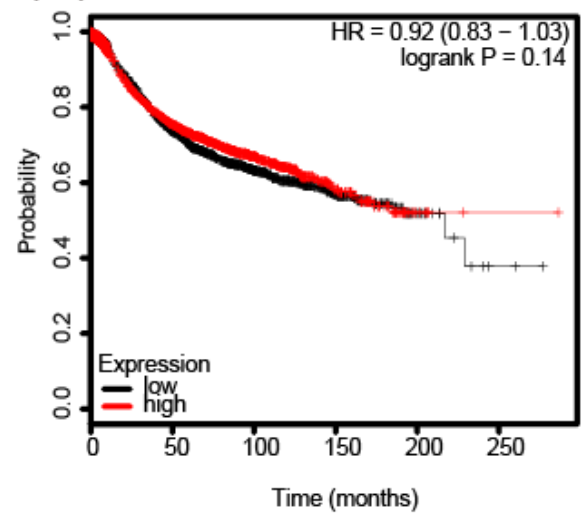

(D)

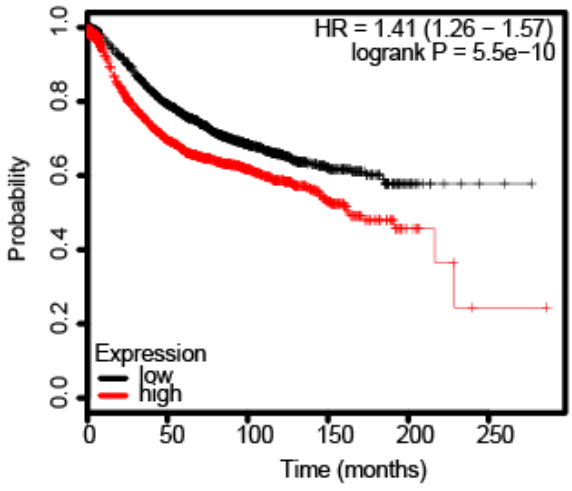

(G) CXCL12 IDO1 PTGS2

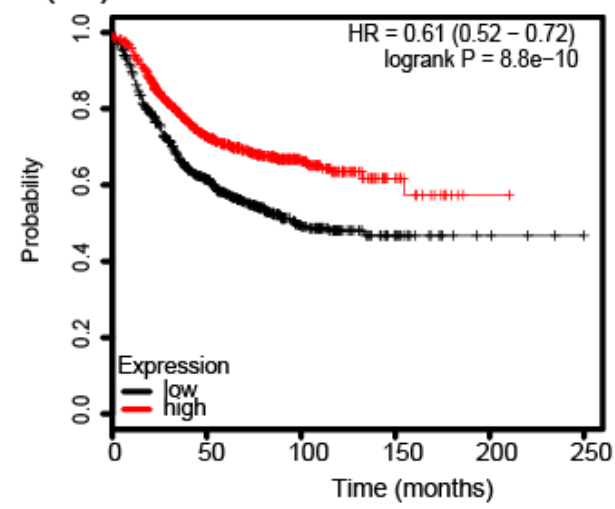

(B)

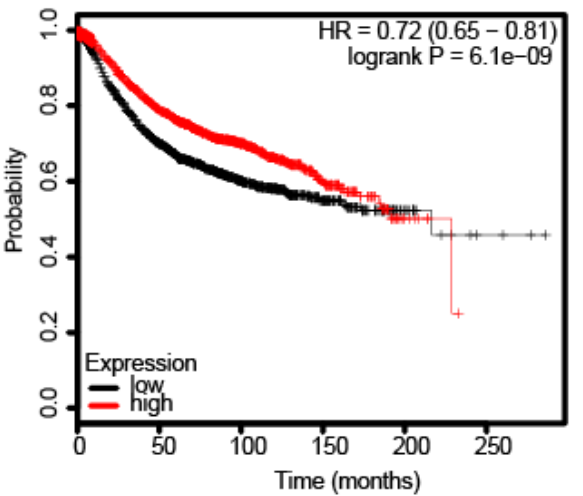

(E)

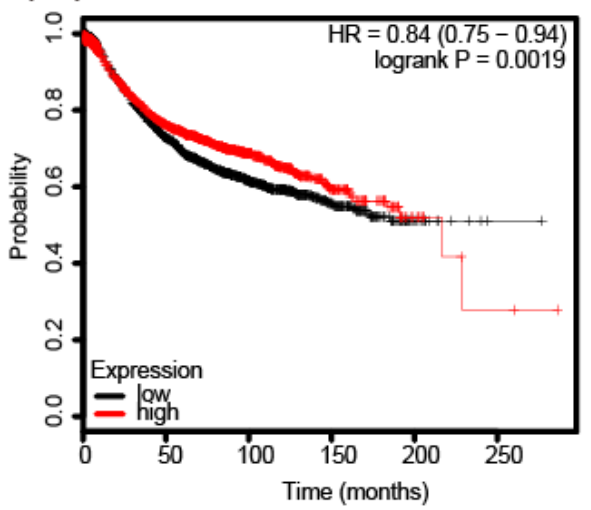

(H)

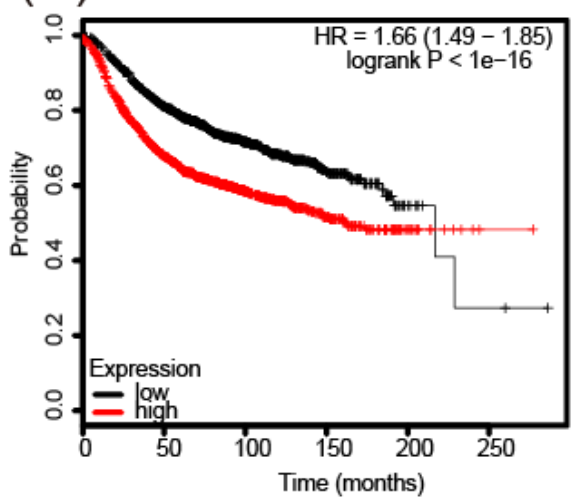

(C) IDO1

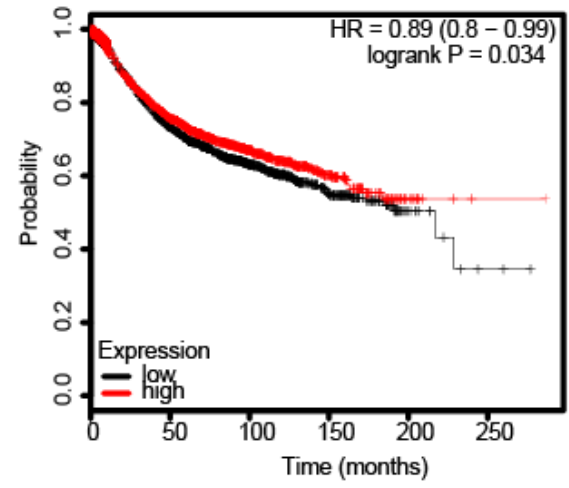

(F)

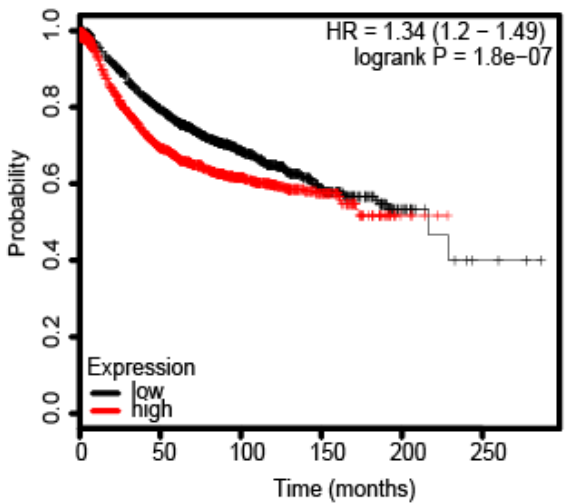




\section{Figure 7}

Immunohistochemical detection of the expression of MIF and VEGFA in a breast cancer tissue microarray.

(A, D) Negative expression (-) in cancer-adjacent normal breast tissue. (B, E) Negative expression in invasive ductal carcinoma. (C, F) Positive expression (+) in invasive ductal carcinoma (original magnification $\times 200$; inset $\times 400$ ).

Cancer adjacent normal breast tissue (-)

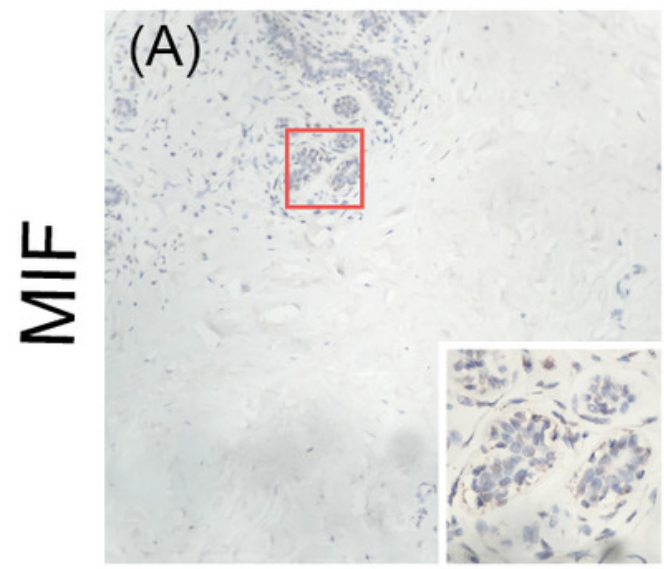

(D)

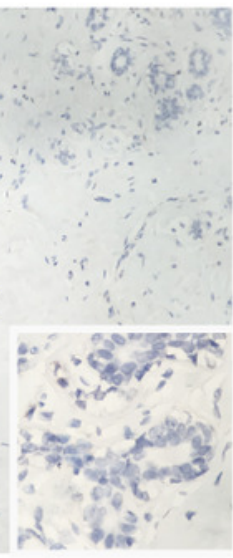

Invasive ductal carcinoma

$(-)$

(B)

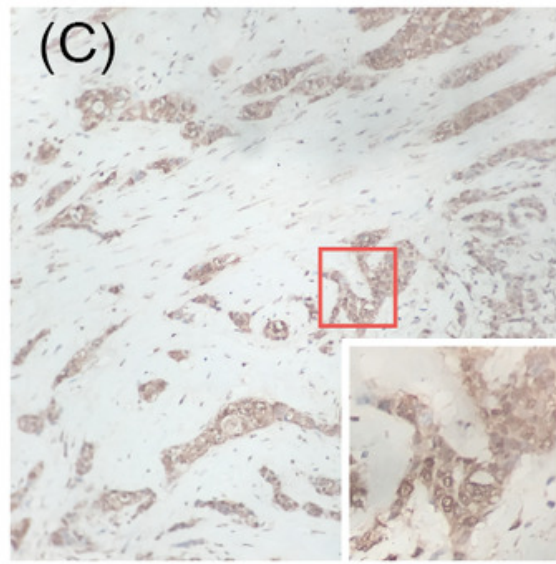

(E)

(F)

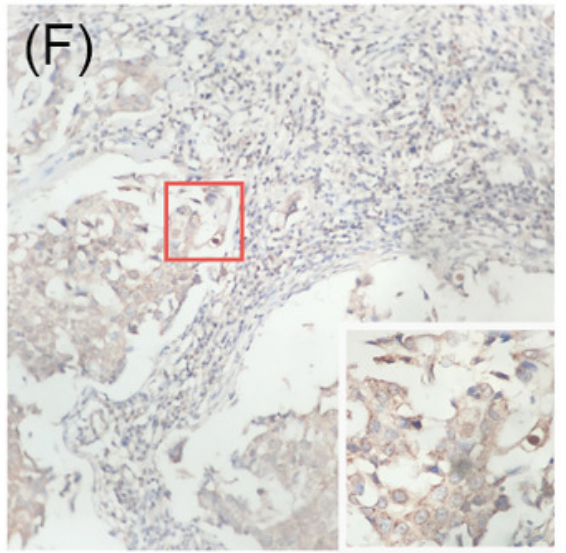


Figure 8

qRT-PCR and western blot results.

(A) qRT-PCR results showed that CD274 and IL8 were upregulated in the basal-like breast cancer cell lines BT549 and MDA-MB-231 ( $p<0.0001)$. Similar to the qRT-PCR results, the western blot analysis (B) indicated that CD274 and IL8 protein were increased in the BT549

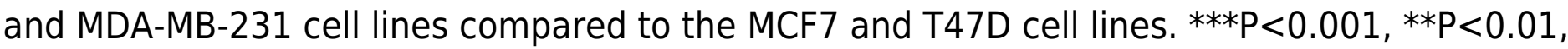
and $* P<0.05$.

(A)
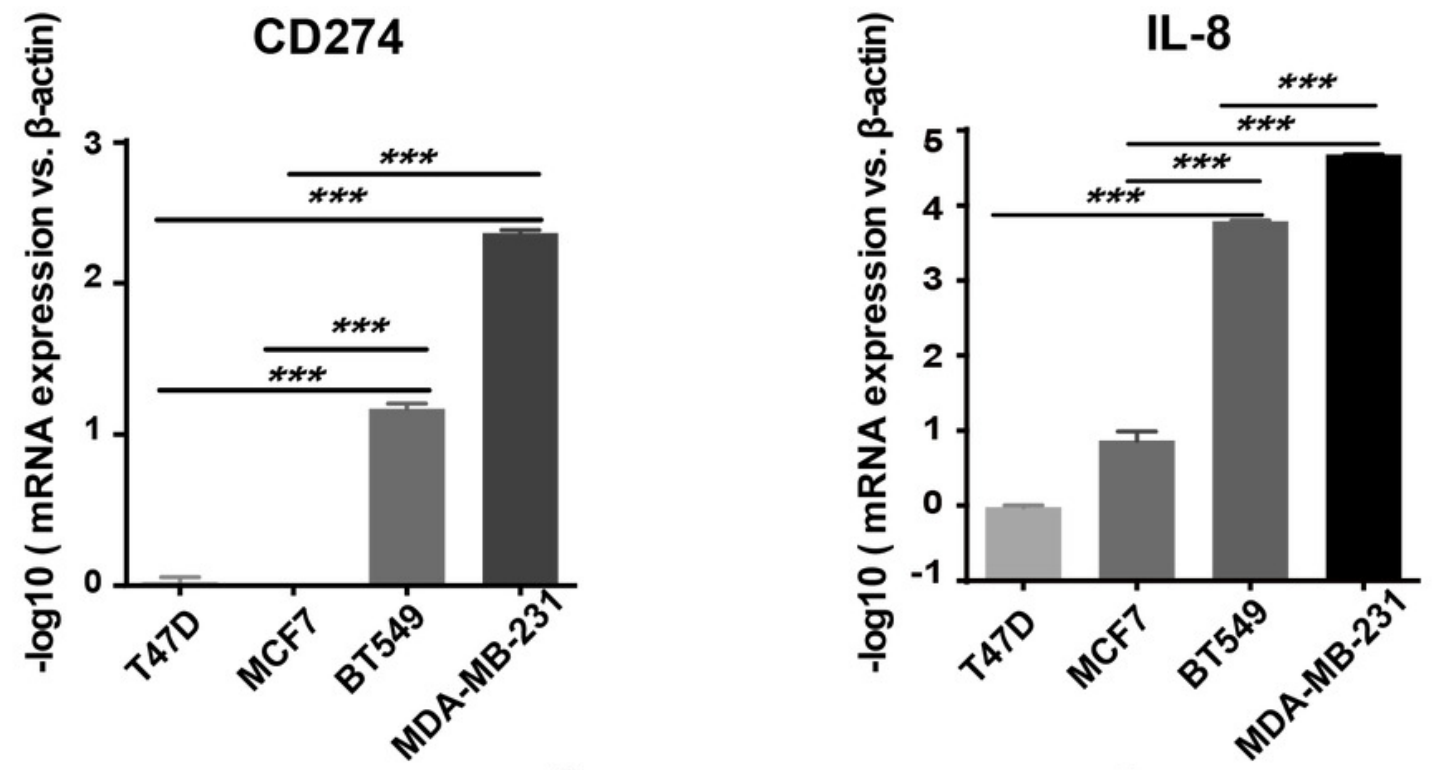

(B)
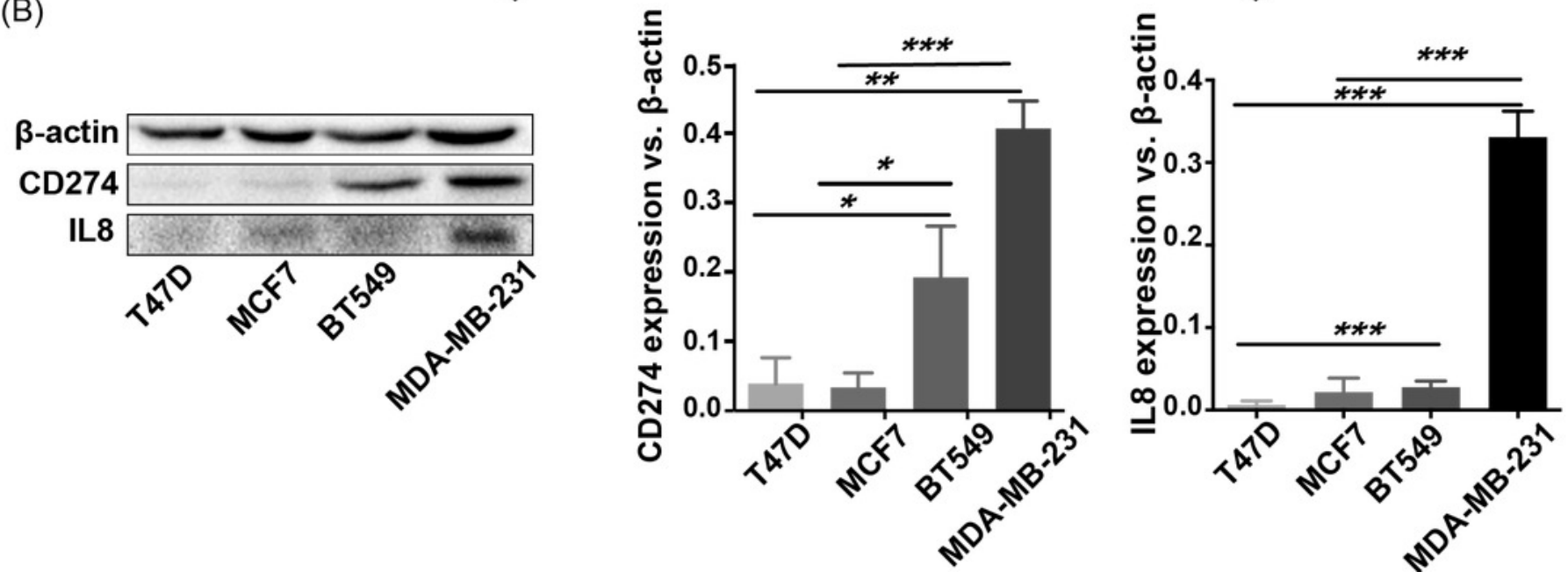


\section{Table $\mathbf{1}$ (on next page)}

TOP $10 \mathrm{GO}$ terms of GO functional annotations for genes in module black 
1 Table 1. TOP $10 \mathrm{GO}$ terms of $\mathrm{GO}$ functional annotations for genes in module black.

\begin{tabular}{|c|c|c|c|}
\hline GO TERM_MF & Count & $\%$ & P-Value \\
\hline GO:0006955 immune response & 72 & 24.91 & $5.94 \mathrm{E}-53$ \\
\hline GO:0002250 adaptive immune response & 30 & 10.38 & $1.38 \mathrm{E}-23$ \\
\hline GO:0050776 regulation of immune response & 31 & 10.72 & $2.30 \mathrm{E}-22$ \\
\hline GO:0060333 interferon-gamma-mediated signaling pathway & 20 & 6.92 & $1.40 \mathrm{E}-18$ \\
\hline GO:0006954 inflammatory response & 37 & 12.80 & 4.16E-18 \\
\hline GO:0050852 T cell receptor signaling pathway & 23 & 7.96 & $1.79 \mathrm{E}-15$ \\
\hline GO:0045087 innate immune response & 34 & 11.76 & 6.47E-14 \\
\hline GO:0050900 leukocyte migration & 20 & 6.92 & $6.71 \mathrm{E}-14$ \\
\hline GO:0031295 T cell costimulation & 17 & 5.88 & $7.59 \mathrm{E}-14$ \\
\hline GO:0019882 antigen processing and presentation & 14 & 4.84 & $2.48 \mathrm{E}-12$ \\
\hline \multicolumn{4}{|l|}{ GO TERM_BP } \\
\hline GO:0042605 peptide antigen binding & 11 & 3.81 & $6.26 \mathrm{E}-12$ \\
\hline GO:0004888 transmembrane signaling receptor activity & 18 & 6.23 & $3.51 \mathrm{E}-08$ \\
\hline GO:0004872 receptor activity & 18 & 6.23 & 4.30E-08 \\
\hline GO:0008009 chemokine activity & 10 & 3.46 & 4.93E-08 \\
\hline GO:0032395 MHC class II receptor activity & 7 & 2.42 & $5.49 \mathrm{E}-08$ \\
\hline GO:0005102 receptor binding & 22 & 7.61 & $1.30 \mathrm{E}-07$ \\
\hline GO:0042288 MHC class I protein binding & 7 & 2.42 & 2.83E-07 \\
\hline GO:0019864 IgG binding & 6 & 2.08 & $3.50 \mathrm{E}-07$ \\
\hline GO:0023026 MHC class II protein complex binding & 6 & 2.08 & 3.11E-06 \\
\hline GO:0003823 antigen binding & 11 & 3.81 & 4.10E-06 \\
\hline
\end{tabular}

2 Note: GO, gene ontology; BP, biological process; MF, molecular function. 
Table 2 (on next page)

TOP 10 clusters of KEGG pathway enrichment analysis for genes in module black 
1 Table 2. TOP 10 clusters of KEGG pathway enrichment analysis for genes in module black

\begin{tabular}{llll}
\hline KEGG_PATHWAY & Count & $\%$ & P-Value \\
\hline hsa05330:Allograft rejection & 19 & 6.57 & $9.37 \mathrm{E}-20$ \\
hsa04612:Antigen processing and presentation & 24 & 8.30 & $2.51 \mathrm{E}-19$ \\
hsa05332:Graft-versus-host disease & 18 & 6.23 & $2.87 \mathrm{E}-19$ \\
hsa05416:Viral myocarditis & 20 & 6.92 & $5.00 \mathrm{E}-17$ \\
hsa04940:Type I diabetes mellitus & 18 & 6.23 & $5.08 \mathrm{E}-17$ \\
hsa05150:Staphylococcus aureus infection & 19 & 6.57 & $3.46 \mathrm{E}-16$ \\
hsa05320:Autoimmune thyroid disease & 18 & 6.23 & $3.69 \mathrm{E}-15$ \\
hsa05152:Tuberculosis & 27 & 9.34 & $2.08 \mathrm{E}-13$ \\
hsa04145:Phagosome & 25 & 8.65 & $2.78 \mathrm{E}-13$ \\
hsa04514:Cell adhesion molecules (CAMs) & 24 & 8.30 & $6.94 \mathrm{E}-13$ \\
\hline
\end{tabular}

2 


\section{Table 3(on next page)}

Relationship between MIF, VEGFA expression level and clinico-pathologic parameters of breast cancer by tissue microarray 
Table3. Relationship between MIF, VEGFA expression level and clinico-pathologic parameters of breast cancer by tissue microarray

\begin{tabular}{|c|c|c|c|c|c|c|c|}
\hline \multirow[t]{2}{*}{ Variable } & \multirow{2}{*}{$\begin{array}{c}\text { Number of } \\
\text { cases }\end{array}$} & \multicolumn{2}{|c|}{ MIF, $100 \%$} & \multicolumn{4}{|c|}{ VEGFA, $100 \%$} \\
\hline & & High & Low & & High & Low & \\
\hline \multicolumn{8}{|c|}{ Pathologic grade } \\
\hline \multirow[t]{2}{*}{1} & 16 & 11 & $5(31.2)$ & & $6(37.5)$ & 10 & \\
\hline & & $(68.8)$ & & 0.397 & & $(62.5)$ & $0.017^{*}$ \\
\hline \multirow[t]{2}{*}{2,3} & 58 & 32 & 26 & & 42 & 16 & \\
\hline & & $(55.2)$ & $(44.8)$ & & $(72.4)$ & $(27.6)$ & \\
\hline \multicolumn{8}{|c|}{ Clinical stage } \\
\hline \multirow[t]{2}{*}{ I } & 17 & $3(17.6)$ & 14 & & 12 & $5(29.4)$ & \\
\hline & & & $(82.4)$ & 0.095 & $(70.6)$ & & 1.000 \\
\hline \multirow[t]{2}{*}{ II, III } & 73 & 30 & 43 & & 51 & 22 & \\
\hline & & $(41.1)$ & $(58.9)$ & & (69.9) & $(30.1)$ & \\
\hline
\end{tabular}

\section{Lymph node status}

No metastasis

78

25

53

48

30

(67.9) $\quad 0.027^{*}$

(61.5)

(38.5) $\quad 0.524$

Metastasis

12

$8(66.7) \quad 4(33.3)$

$9(75.0) \quad 3(25.0)$

1 The total number of samples in pathologic grade does not equal 90, as some samples are not included in any given

2 grades. ${ }^{*} p<0.05$

3

4 